\title{
An Ensemble Machine Learning Model based on Multiple Filtering and Supervised Attribute Clustering Algorithm for Classifying Cancer Samples
}

\author{
Shilpi Bose ${ }^{\text {Corresp., } 1}{ }^{\text {, Chandra Das }}{ }^{1}$, Abhik Banerjee ${ }^{1}$, Kuntal Ghosh $^{2}$, Matangini Chattopadhyay ${ }^{3}$, Samiran \\ Chattopadhyay ${ }^{4}$, Aishwarya Barik ${ }^{1}$ \\ 1 \\ ${ }^{1}$ Department of Computer Science and Engineering, Netaji Subhash Engineering College, Kolkata, West Bengal, India \\ Machine Intelligence Unit \& Center for Soft Computing Research, Indian Statistical Institute, Kolkata, West Bengal, India \\ 3 School of Education Technology, Jadavpur University, Kolkata, West Bengal, India \\ 4 Department of Information Technology, Jadavpur University, Kolkata, West Bengal, India \\ Corresponding Author: Shilpi Bose \\ Email address: shilpi.bose@nsec.ac.in
}

Background: Machine learning is one kind of machine intelligence technique that learns from data and detects inherent patterns from large, complex datasets. Due to this capability, machine learning techniques are widely used in medical applications, especially where large-scale genomic and proteomic data are used. Cancer classification based on bio-molecular profiling data is a very important topic for medical applications since it improves the diagnostic accuracy of cancer and enables a successful culmination of cancer treatments. Hence, machine learning techniques are widely used in cancer detection and prognosis.

Methods: In this article, a new ensemble machine learning classification model named Multiple Filtering and Supervised Attribute Clustering algorithm based Ensemble Classification model (MFSAC-EC) is proposed which can handle class imbalance problem and high dimensionality of microarray datasets. This model first generates a number of bootstrapped datasets from the original training data where the oversampling procedure is applied to handle the class imbalance problem. The proposed MFSAC method is then applied to each of these bootstrapped datasets to generate sub-datasets, each of which contains a subset of the most relevant/informative attributes of the original dataset. The MFSAC method is a feature selection technique combining multiple filters with a new supervised attribute clustering algorithm. Then for every sub dataset, a base classifier is constructed separately, and finally, the predictive accuracy of these base classifiers is combined using the majority voting technique forming the MFSAC-based ensemble classifier. Also, a number of most informative attributes are selected as important features based on their frequency of occurrence in these sub-datasets.

Results: To assess the performance of the proposed MFSAC-EC model, it is applied on different highdimensional microarray gene expression datasets for cancer sample classification. The proposed model is compared with well-known existing models to establish its effectiveness with respect to other models. From the experimental results, it has been found that the generalization performance/testing accuracy of the proposed classifier is significantly better compared to other well-known existing models. Apart from that, it has been also found that the proposed model can identify many important attributes/biomarker genes. 


\section{An Ensemble Machine Learning Model based on}

${ }^{2}$ Machine Intelligence Unit \& Center for Soft Computing Research, Indian Statistical Institute, Shilpi Bose ${ }^{1}$, Chandra Das ${ }^{1}$, Abhik Banerjee ${ }^{1}$, Kuntal Ghosh ${ }^{2}$, Matangini Chattopadhyay ${ }^{3}$, Samiran Chattopadhyay ${ }^{4}$, Aisarya Barik ${ }^{1}$ Kolkata, West Bengal, India

Corresponding Author:

Shilpi Bose

Department of CSE, Netaji Subhash Engineering College, Panchpota, Garia, Kolkata, 700152, West Bengal, India

\section{Abstract}

Background: Machine learning is one kind of machine intelligence technique that learns from data and detects inherent patterns from large, complex datasets. Due to this capability, machine learning techniques are widely used in medical applications, especially where large-scale genomic and proteomic data are used. Cancer classification based on bio-molecular profiling data is a very important topic for medical applications since it improves the diagnostic accuracy of cancer and enables a successful culmination of cancer treatments. Hence, machine learning techniques are widely used in cancer detection and prognosis.

Methods: In this article, a new ensemble machine learning classification model named Multiple Filtering and Supervised Attribute Clustering algorithm based Ensemble Classification model (MFSAC-EC) is proposed which can handle class imbalance problem and high dimensionality of microarray datasets. This model first generates a number of bootstrapped datasets from the original training data where the oversampling procedure is applied to handle the class imbalance problem. The proposed MFSAC method is then applied to each of these bootstrapped datasets to generate sub-datasets, each of which contains a subset of the most relevant/informative attributes of the original dataset. The MFSAC method is a feature selection technique combining multiple filters with a new supervised attribute clustering algorithm. Then for every sub dataset, a base classifier is constructed separately, and finally, the predictive accuracy of these base classifiers is combined using the majority voting technique forming the MFSAC-based ensemble classifier. Also, a number of most informative attributes are selected as important features based on their frequency of occurrence in these sub-datasets. 
40 Results: To assess the performance of the proposed MFSAC-EC model, it is applied on different 41 high-dimensional microarray gene expression datasets for cancer sample classification. The 42 proposed model is compared with well-known existing models to establish its effectiveness with 43 respect to other models. From the experimental results, it has been found that the generalization 44 performance/testing accuracy of the proposed classifier is significantly better compared to other 45 well-known existing models. Apart from that, it has been also found that the proposed model can identify many important attributes/biomarker genes.

\section{Introduction}

Cancer is one of the most fatal diseases around the globe (Tabares-Soto 2020, Hambali 2020). According to the World Health Organization report, Cancer is marked as the second most deadly disease and an estimated 9.7 million deaths around the world in 2018 have occurred due to this signature disease (Hambali 2020). Generally, one in every 6 deaths all over the world, occurs due to cancer. So, within 2030, the number of new cancer patients per year will increase approximately by 25 million (Hambali 2020, NIH 2019). Although several advanced techniques are already developed for the detection of cancer, the proper prognosis of cancer patients, till date, is very poor and the survival rate is also very low (Tabares-Soto 2020, Hambali 2020, Konstantina 2015). It has been already found that for very accurate cancer sample classification or prediction, adequate information is not available from the clinical, environmental, and behavioral characteristics of patients (Kourou 2015, Hambali 2020, Tabares-Soto Reinel 2020). Recently, due to different types of bio-molecular data analysis, several genetic disorders with different biological characteristics have been revealed which are very helpful for early identification and prognosis of cancer and also to discern the responses for different types of treatment (Colozza2005, Greller 1999, Li 2018, Liu2011, Pilling 2017, Su 2001, Swan 2013). With the rapid advancements in genomic, proteomic, and imaging high-throughput technologies (Colozza2005, Greller 1999, Li 2018, Liu2011, Pilling2017, Su 2001, Swan 2013), now it is possible to accumulate huge amount (in the order of thousands) of different bio-molecular information of patients. Using this huge amount of information, researchers have been trying to develop more advanced techniques for early detection and proper prognosis of cancer, and also to improve cancer therapy for improvement of patients' survival rate. To analyze this huge amount of information, lab-based approaches are not adequate as these methods are costly and time-consuming. So, computational or in-silico methods like statistical methods, machine learning, deep learning, etc. have been being used extensively in this field. It is well-known fact that in cancer-causing cells, gene expression is either overexpressed or under expressed (Tabares-Soto 2020). So, measurement of gene expression in cancer cells can give adequate information to improve cancer diagnostic procedures. Nowadays, different developing countries have been using this procedure for cancer sample detection. It is already known that using DNA microarray technology it is possible to measure the expression level of a numerous number of genes for a single experiment/sample simultaneously. The outcome of DNA microarray technology is a gene expression data matrix. This matrix carries information 
80 about the expression level of a huge number of genes for a limited number of samples (such as 81 diseased patient samples and normal samples). The presence of the limited number of samples in 82 this data matrix is due to the lack of availability of samples. So, based on information of gene 83 expression data matrix, cancer sample classification is one of the essential tasks in the field of 84 cancer research (Chin 2016, Dashtban 2017, Ding 2005, Elyasigomari 2017, Furey2000, Golub 85 1999, Nada 2019, Tabares-Soto 2020).

86 Using computational or in-silico approaches, gene expression-based cancer sample classification 87 task has been reviewed extensively in different papers (Chin 2016, Dashtban2017, Ding 2005, 88 Elyasigomari 2017, Furey2000, Golub 1999, Nada 2019, Tabares-Soto 2020). However, the 89 main difficulties in the sample classification task arise due to several factors. Firstly, in these 90 data sets, a substantially small number of samples is available (generally in the order of 91 hundreds) compared to the availability of a huge number of genes (generally in the order of 92 thousands) (Chin 2016, Hambali 2020, Nada 2019). For sample classification, genes are treated as features/attributes. So, the high-dimensional gene space is an overhead for most classification algorithms. Secondly, only a very few genes are informative (differentially expressed) and the rest of the section is non-informative (noisy) (Chin 2016, Hambali 2020, Nada 2019) for sample classification and responsible for degrading the classifier's performance. Gene dimension reduction by identification of informative genes as biomarkers can improve the classification accuracy of classifiers. Apart from the improvement of classification accuracy, the identification of informative biomarkers (here, informative genes) has great prospects from a biomedical point of view. These are beneficial for finding the biological reason for a disorder, assessing disease risk, and developing therapeutic targets. The third problem arises due to the small sample size which creates an overfitting problem in classifier construction. Another problem that degrades classifier performance is the sample class imbalance problem. This problem occurs due to the presence of more instances/samples of one class (majority class) with respect to other class(es) (minority class) in a dataset. A fairly large number of works have been already developed for sample classification. These works are divided into two categories. In the first category (Chin 2016, Hambali 2020, Nada 2019), the major emphasis is given to the selection of relevant genes for the reduction of feature space. Then based on this reduced feature space, predictive/classification accuracy of the samples is measured using different existing single classification models like naïve Bayes, support vector machine, relevance vector machine, K-nearest neighbor, decision tree, logistic regression, etc. As gene selection is a feature selection task, so based on feature selection techniques, these methods are divided into different categories. These are (1) filter methods (2) wrapper methods (3) embedded methods and (4) hybrid methods. Before we mention the second category of classification methods, let us first elaborate on the first category methods one by one. Filter methods (Chin 2016, Hambali 2020, Nada 2019) select a subset of features without taking any information from any classification model. These methods select features that are differentially expressed with respect to sample class labels. The filter methods rank individual features according to their class discrimination power based on some statistical score function 
120

121

122

123

124

125

126

127

128

129

130

131

132

133

134

135

136

137

138

139

140

141

142

143

144

145

146

147

148

149

150

151

152

153

154

155

156

157

158

159

and then select a number of high-ranked features to form a reduced and relevant feature subset. The popular statistical score functions used in filter methods are Fisher's score, Signal to Noise ratio (SNR), correlation coefficient, mutual information, Relief (Das 2019), etc. Filter methods are computationally simple, fast, and unbiased in favor of any specific classifier as these methods do not consider any knowledge from any classifier at the feature selection phase. The drawback of filter methods is that the number of selected features is based solely on the trial-error method. Wrapper methods (Chin 2016, Hambali 2020, Nada 2019), on the other hand, judge discrimination capability of a feature subset using classification error rate or prediction accuracy of a classifier as the feature evaluation function. It selects the most discriminative feature subset via minimizing the classification error rate or maximizing the classification accuracy of a classifier. The wrapper methods generally achieve better classification accuracy than the filter methods because the selection of feature subset is classifier-dependent. One drawback of these methods is that these are biased to used classifiers and another drawback is that these are computationally more expensive than the filter methods as generation of the best feature subset for the high-dimensional dataset is an NP-complete problem. Due to these reasons, these methods are not applicable for high-dimensional datasets.

In Embedded methods (Chin 2016, Hambali 2020, Nada 2019), the optimal feature subset is selected through the unique learning procedure of a specific classifier at the time of classifier construction. Actually, in these methods, the optimal feature subset selection part is embedded as part of classifier construction. These methods are faster than wrapper methods but are biased to the specific classifier. In embedded approaches, the feature selection process is specific for a particular classifier and is not applicable to other classifiers. These are also computationally expensive. Due to these reasons for high-dimensional datasets, these methods are not applicable. On the other hand, recently hybrid feature selection methods (Chin 2016, Hambali 2020, Nada 2019) are also developed. In hybrid methods, different category-based methods are combined to take advantage of all of these methods for improving classification accuracy. Apart from these methods, clustering techniques (Chin 2016, Hambali 2020) are also used for feature selection purposes. Clustering techniques divide the data space in such a manner that objects in the same cluster are similar while in different clusters they are dissimilar. For the feature selection task, clustering methods (famous as attribute clustering in feature selection domain) (Au 2005) divide the features into several distinct clusters and then reduce the feature dimension by selecting a small number of significant features from each cluster. A lot of unsupervised gene (attribute) clustering algorithms (Au2005, Chin 2016, Hambali 2020) are already developed for this task. However, these methods are unsuccessful to find informative functional groups of genes for sample classification as in clustering genes, no supervised information from sample classes is considered (Au2005, Chin 2016, Hambali 2020). So, scientists have developed a number of supervised gene (attribute) clustering algorithms (Dettling 2002, Hastie 2000, Hastie 2001, Maji 2012) in which genes are grouped using supervised information from sample classes and a reduced gene set is formed via selecting the most informative genes from each cluster. 
160

161

162

163

164

165

166

167

168

169

170

171

172

173

174

175

176

177

178

179

180

181

182

183

184

185

186

187

188

189

190

191

192

193

194

195

196

197

198

199

All the above-mentioned variants deliver comparable feature selection and classification accuracy. Quite often this type of classification models with only a few genes and with a limited number of training samples can classify the majority of training samples correctly, but the generalization capability of such classification models cannot be guaranteed (Bolo2012, Ghorai 2011, Nagi 2013, Wang 2006, Wang 2012, Yang 2010). So, the most important task for a medical diagnosis system is to improve the classification accuracy of unknown samples (generalization performance) which cannot be solved by this type of classification model. Apart from this problem, the microarray data is related to several uncertainties due to fabrication, hybridization, and image processing procedure in microarray technology. These uncertainties introduce various types of noise in microarray data. Due to the presence of these uncertainties with a limited number of training samples, the conventional machine learning approaches face challenges to develop reliable classification models.

To overcome the above-mentioned problems, it is therefore essential to develop general approaches and robust methods. In this regard, researchers are motivated to develop the second category-based model. These are the different robust ensemble classification models (Bolo2012, Ghorai 2011, Nagi 2013, Osareh 2013, Wang 2006, Wang 2012, Yang 2010) which can overcome small sample size problems and are capable of removing uncertainties of gene expression data.

Ensemble methods (Dietterich 2000) are a class of machine learning technique which combines multiple base learning algorithms to produce one optimal predictive model. Ensemble classification model refers to a group of individual/base classifiers that are trained individually on the trained dataset in a supervised classification system and finally, an aggregation method is used to combine the decisions produced by the base classifiers. These ensemble classification models have the potential to alleviate the small sample size problem by applying multiple classification models on the same training data or on bootstrapped samples (sampling with replacement) of the training data to decrease the chance of overfitting in the training data. In this way, the training dataset is utilized more efficiently, and as a consequence, the generalization ability is improved.

Although different category-based ensemble classification models exist in the literature but these ensemble models are not capable of addressing all the above-mentioned problems (small sample size, high dimensional feature space, and sample class imbalance problem) related to microarray data.

In this regard, here a new Multiple Filtering and Supervised Attribute Clustering algorithm-based ensemble classification model named MFSAC-EC is proposed. In this model, first, a number of bootstrapped versions of the original training dataset are created. At the time of the creation of bootstrapped versions, an oversampling technique ( Blaszczynski 2013 ) is adopted to solve the class imbalance problem. For every bootstrapped dataset a number of sub-datasets (each with a subset of genes) are generated using the proposed MFSAC method. The MFSAC is a hybrid method combining multiple filters with a new supervised attribute clustering method. Then for every sub dataset, a base classifier is constructed. Finally, based on the prediction accuracy of all 
200 these base classifiers of all sub-datasets for all bootstrapped datasets an ensemble classifier (EC)

201 is formed using the majority voting technique.

202 The novelty of the proposed MFSAC-EC model is that here the emphasis is given

203 simultaneously on the high dimensionality problem of gene expression data, small sample size

204 problem as well as the class imbalance problem. All of these problems at the same time are not

205 considered in any existing ensemble classification model. First of all, due to the use of

206 bootstrapping method with a class balancing strategy, the proposed model can handle a small

207 sample size and overfitting problem. Secondly, in MFSAC, different filter methods are used with

208 their unique characteristics. So, different characteristics-based relevant gene subsets are selected

209 via different filters to form different sub-datasets from every bootstrapped dataset. Finally, every

210 gene subset is modified using a supervised attribute clustering algorithm. In this way, the high-

211 dimensionality problem of gene expression data is handled here. Apart from this, from the

212 MFSAC generated sub-datasets, the frequency of occurrence is counted for every gene and

213 informative genes are ranked accordingly. The prediction capability of the proposed model is

214 experimented with over different microarray datasets and compared with the existing well-

215 known models. Experimental outputs demonstrate the superiority of the proposed model over

216 existing models.

\section{Materials \& Methods}

218 The proposed MFSAC-EC model is composed of different filter score functions, a new

219 supervised attribute clustering method, and an ensemble classification method. In the following

220 subsections, first, a brief overview is given on different filter score functions and then the

221 proposed MFSAC-EC model is described.

\section{Preliminaries}

223 In this paper, a data set (here, a microarray gene expression data set) is represented by a data

224 matrix, $K_{U \times V}$, with $U$ data objects (samples) and $V$ features (genes). The set of objects or

225 samples is represented as $\mathrm{E}=\left\{E_{1}, E_{2}, \ldots . ., E_{S}, \ldots E_{U}\right\}$ while the set of genes is represented as $G=\{$

226 $\left.G_{1}, G_{2}, \ldots \ldots, G_{t}, \ldots . G_{V}\right\}$. Here, each sample is a $V$-dimensional feature vector containing $V$ number of gene expression values. Similar way, every gene is a $U$-dimensional vector containing $U$ number of sample values. Here, $C_{U \times 1}$ is a class vector representing the associated class label for every sample. The class label is taken from a set $D C=\left\{d_{1}, d_{2}, \ldots ., d_{j}, \ldots d_{N}\right\}$ with $N$ distinct class labels.

\section{Brief overview of Filter score functions used in MFSAC}

The filter score functions used in the proposed MFSAC-EC model are modified Fisher score (Gu 2011), modified T-test (Zhou 2007), Chi-square (Das 2019), Mutual information (Das 2019), Pearson correlation coefficient (Leung 2010), SNR(Leung 2010) and Relief-F (Das 2019). A summary of these 7 filters used in the MFSAC-EC model is given in the supplemental Table S1. In the proposed MFSAC-EC model, initially, bootstrapping (sampling with replacements) with a class balancing procedure of samples is applied on training dataset $K$ to create $D$ number of different bootstrapped versions from the training dataset. Here, every bootstrapped dataset with 
$239 U$ samples is formed by random sampling with replacements $U$ times from the original dataset $K$.

240 After that oversampling procedure is applied to each minority class to achieve data balance.

241 Oversampling consists of increasing the minority class instances by their random replication to

242 exactly balance the cardinality of the minority and majority classes in each bootstrapped dataset.

243 Due to oversampling each bootstrapped dataset will contain more instances than the original

244 dataset.

245 The MFSAC method of the MFSAC-EC model, which is an integration of multiple filters and a

246 new supervised attribute (gene) clustering method, is applied on every newly created

247 bootstrapped $\left(B K_{l}\right)$ training dataset. The proposed MFSAC method first calculates the class relevance score of every gene present in the bootstrapped training dataset using each filter score function $\left(F T_{x}\right), x=1$ to 7 mentioned above. Then for each filter score function, a sub dataset $S$ $D_{l x}$ with a gene subset $\left(G S_{l x}\right)$ is created by selecting a predefined number (let $P$ ) of the most relevant genes from the full gene set $G$. So, $\left|G S_{l x}\right|=P$. After that on every gene subset $\left(G S_{l x}\right)$ of every sub dataset $S D_{l x}$, the SAC (Supervised Attribute Clustering) method is applied and a set of clusters $C G S_{l x}$ and corresponding cluster representatives (considered as modified features) are

254

255

256

257

258

259

260

261

262

263

264

265

266

267

268

269

270

271

272

273

formed. Finally, $Q$ numbers of most relevant cluster representatives are selected as modified features and a reduced sub dataset $R S D_{l x}$ of the sub dataset $S D_{l x}$ is formed. How the SAC method works on $G S_{l x}$ of every sub dataset $S D_{l x}$ is discussed below.

For any sub dataset $S D_{l x}$, the SAC method starts by selecting the gene from the subset $\left(G S_{l x}\right)$ with the highest $F T_{x}$ value. Let gene $G_{l i} \in G S_{l x}$ with the highest $F T_{x}$ value be selected as the first member (let $F T_{x}\left(G_{l i}, C\right)=A$ ) and it also becomes the initial cluster representative $R\left(R=G_{l i}\right)$ ) of the first cluster $C_{1} G S_{l x}$ and $G_{l i}$ is deleted from $G S_{l x}$. In effect, $G_{l i} \in C_{1} G S_{l x}$, and $G S_{l x}=G S_{l x}$ $-\left\{G_{l i}\right\}$ and so $F T_{x}(R, C)=A$. This cluster is then grown up in parallel with the cluster representative refinement process which is described next. In this process, the gene (let $G_{l m}$ ) with next highest $F T_{x}$ value is taken from $G S_{l x}$ subset and is merged with the current cluster representative $R$. The merging is done in two ways. Firstly the expression profile of $G_{l m}$ is directly added with $R$ and a temporary augmented representative $T R^{+}$is formed and its $F T_{x}$ value (let $B_{1}$ ) is calculated. The second one is that the sign-flipped value of the expression profile of $G_{l m}$ is added with $R$ and another temporary augmented representative $T R^{-}$is formed and its $F T_{x}$ value (let $\left.B_{2}\right)$ is calculated. If $F T_{x}\left(T R^{+}, C\right) \geq F T_{x}\left(T R^{-}, C\right)$ that is $B_{1} \geq B_{2}$ then $T R^{+}$is chosen else $T R^{-}$is chosen. Let $T R^{+}$is chosen. Now if $F T_{x}\left(T R^{+}, C\right)>F T_{x}(R, C)$ then $R=T R^{+}$otherwise, $R$ is unaltered. Similar way if $T R^{-}$is chosen and if $F T_{x}\left(T R^{-}, C\right)>$ $F T_{x}(R, C)$ then $R=T R^{-}$otherwise, $R$ remains unchanged. If $R$ is modified then the gene $G_{l m}$ is included in the cluster and $G_{l m}$ is deleted from $G S_{l x}$. In effect, $G_{l m} \in C_{1} G S_{l x}$, and $G S_{l x}=G S_{l x}-$ $\left\{G_{l m}\right\}$. So, the next chosen gene is included in the current cluster if it improves the class 
274 relevance value of the current cluster representative. The merging process is described in Figure 2751.

276 Here g0 represents the current cluster representative $(R)$ and its class relevance score $\left(\left(F T_{x}, R\right)\right.$,

277 here Pearson score), is shown. Now among all the genes g1, g2, g3, g4, and g5, the Pearson score

278 of $\mathrm{g} 1$ is the highest. So, $\mathrm{g} 1$ is chosen for the merging process. Then $\mathrm{g} 1$ is added with $R$ to create

279 the temporary augmented representative $\left(T R^{+}=R+g 1\right)$ and also its sign-flipped value is

280 added with the $R$ to form the temporary augmented representative $\left(T R^{-}=R-g 1\right)$. The Pearson

281 score of $T R^{+}$is greater than the Pearson score of $T R^{-}$, so $T R^{+}$is chosen. Now the Pearson

282 score of $T R^{+}$is greater than the Pearson score of $R$, so $T R^{+}$is considered as the current cluster

283 representative and $R=T R^{+}$. This process is continued for all other genes. Now, $\mathrm{g} 3$ is chosen as

284 it is the gene with the next highest Pearson value. $\mathrm{g} 3$ and its sign-flipped value are added

285 individually with current cluster representative $R$ to form $T R^{+}=R+g 3$ and $T R^{-}=R-g 3$

286 respectively. In this case, Pearson score of $T R^{-}$is greater than the Pearson score of $T R^{+}$. So,

$287 T R^{-}$is chosen. Then Pearson score of $T R^{-}$is Checked with the Pearson score of $R$ and here

288 Pearson score of $T R^{-}$is greater than the Pearson score of $R$. So, $T R^{-}$is considered as current

289 cluster representative and $R=T R^{-}$. In this way, cluster representative is refined. This process is

290 repeated for every member of $G S_{l x}$ subset.

291 After the formation of the first cluster and its corresponding augmented representative, $R$ is

292 assigned to $A R_{l x 1}$ that means $A R_{l x 1}=R$, and the supervised clustering process is repeated to

293 form the second cluster with the gene (let $G_{l z}$ ) with next highest $F T_{x}$ value from $G S_{l x}$ subset. In

294 this way a set of clusters $C G S_{l x}=\left\{C_{1} G S_{l x}, C_{2} G S_{l x}, \ldots . ., C_{k} G S_{l x}, \ldots.\right\}$ and their corresponding

295 augmented cluster representatives $A R_{l x}=\left\{A R_{l x 1}, \ldots \ldots \ldots, A R_{l x k}, \ldots ..\right\}$ are formed. After that $Q$

296 number of most powerful augmented cluster representatives are chosen (as modified features)

297 according to their $F T_{x}$ value from the generated clusters and with these $Q$ number of modified

298 features, a reduced sub dataset $R S D_{l x}$ of sub dataset $S D_{l x}$ is formed.

299 In this way, for every bootstrapped version $\left(B K_{l}\right)$ of the training dataset, 7 number of $R S D_{l x}$ sub-

300 datasets are created and for every $R S D_{l x}$ an individual classifier is constructed using any existing

301 classifier and finally, an ensemble classifier (EC) is formed by combining all these classifiers of

302 all bootstrapped versions using the majority voting technique. To classify every sample using

303 this ensemble classifier, each classifier votes or classifies the sample for a particular class, and

304 the class for which the highest number of votes is obtained is considered as the output class.

305 MFSAC method based Informative Attribute Ranking

306 For every gene (feature/attribute), the frequency of occurrence that means the total number of

307 times it appears in all sub-datasets generated by the MFSAC method for all bootstrapped

308 versions is calculated. Then according to their frequency of occurrence, those genes are ordered

309 or ranked. The top-ranked genes with the highest occurrence frequency are considered the most

310 informative cancer-related genes. 
311 The block diagram of the proposed MFSAC-EC model is shown in Figure 2, while the block 312 diagram of the MFSAC method is shown in Figure 3. The algorithm of the proposed model is 313 described below.

314

315

316

317

318

319

320

321

322

323

324

325

326

327

328

329

330

331

332

333

334

335

336

337

338

339

340

341

342

343

344

345

346

347

47

\section{Algorithm: MFSAC-EC}

Input: $\mathrm{A} \mathrm{K}_{U \times V}$ data matrix (here, gene expression data matrix) containing $U$ number of data objects (here, cancer samples) and $V$ number of attributes (here, genes).

Output: An ensemble classifier MFSAC-EC is formed to classify test samples. From MFSAC generated sub-datasets, informative genes are selected according to their rank. Every gene is ranked according to its frequency of occurrence.

\section{Definitions:}

$\mathrm{E}=\left\{E_{1}, E_{2}, \ldots ., E_{S}, \ldots E_{U}\right\}$ is the set of objects or samples of $\mathrm{K}_{U \times V}$ data matrix. Every sample $E_{s}$ is a $V$ dimensional vector.

$G=\left\{G_{1}, G_{2}, \ldots \ldots, G_{t}, \ldots G_{V}\right\}$ is the set of features or genes of $\mathrm{K}_{U \times V}$ data matrix. Every gene $G_{t}$ is a $U$ dimensional vector. $B K=\left\{B K_{1}, B K_{2}, \ldots, B K_{l}, \ldots . ., B K_{D}\right\}$ is a set of the bootstrapped version of the original training dataset. In every bootstrapped dataset the number of samples varies from the original dataset but the number of features is the same as the original dataset.

$C_{U \times 1}$ is a class vector representing the associated class label for every sample. For a data matrix $N$ distinct class labels exist and class labels are taken from a set $D C=\left\{d_{1}, d_{2}, \ldots . ., d_{k}, \ldots d_{N}\right\}$. $F T_{x}\left(G_{t}, C\right)$ is $x^{\text {th }}$ filter score function which returns the class relevance value of $G_{t}$ gene with respect to class vector $C$ using $F T_{x}$ score function, for $x=1$ to 7 as 7 represents the total number of filtering score functions used here. $G S_{l x}\left(G S_{l x}=P\right)$ is a set of top-ranked genes of $G$ selected using $F T_{x}$ score function and $S D_{l x}$ is corresponding sub dataset of $B K_{l}$. Here $S D_{l x}$ is a data matrix containing $P$ number of genes. $C G S_{l x}=\left\{C_{1} G S_{l x}, C_{2} G S_{l x}, \ldots . ., C_{k} G S_{l x}, \ldots\right\}$ and $A R_{l x}=\left\{A R_{l x 1}, \ldots \ldots . ., A R_{l x k}, \ldots . ..\right\}$ are the set of clusters and corresponding cluster representatives respectively generated from the corresponding subset $G S_{l x}$ of $S D_{l x}$. Here every $A R_{l x k}$ is a vector.

$\mathrm{TR}^{+}, \mathrm{TR}^{-}, \mathrm{R}$ are vectors similar to a gene vector. $R S D_{l}=\left\{R S D_{l 1}, R S D_{l 2}, \ldots \ldots \ldots, R S D_{l x}, \ldots \ldots . R S D_{l 7}\right\}$ is a set of sub-datasets each containing $Q$ number of most relevant cluster representatives formed for every bootstrapped dataset $B K_{l}$. $C F_{l}=\left\{I C_{l 1}, I C_{l 2}, \ldots \ldots ., I C_{l x}, \ldots \ldots I C_{l 7}\right\}$ is a set of classifiers formed for every bootstrapped dataset.
1. Create $D$ number bootstrapped version of training dataset $K$.

2. For Every bootstrapped dataset $B K_{l}$ repeat step 3

3. Repeat for $x=1$ to 7
A. Repeat for $t=1$ to $V$ 
348

349

350

351

352

353

354

355

356

357

358

359

360

361

362

363

364

365

366

367

368

369

370

371

372

373

374

375

376

377

378

379

380

381

382

a) Calculate class relevance score $F T_{x}\left(G_{t}, C\right)$ of $G_{t}$ gene, where $G_{t} \in G$, with respect to class vector $C$

B. Select $P$ number of top-ranked genes from $G$ based on $F T_{x}$ score function and form $G S_{l x}$ gene subset with corresponding $S D_{l x}$ sub dataset

C. Set $k=0$

D. Repeat until $G S_{l x}=\varnothing$

a) Set $k=k+1$

b) Set $A R_{l x k}=0, R=0$, and $i=0$

c) Select the gene (let $G_{l i}$ ) whose $F T_{x}$ score value is maximum among all genes of $G S_{l x}$ and set $R=G_{l i}$

d) Add $G_{l i}$ to $C_{k} G S_{l x}$, and delete $G_{l i}$ from $G S_{l x}$

e) Set count $=1$

f) Repeat for $j=1$ to $\left|G S_{l x}\right|$

I. Compute first augmented representatives $T R^{+}$by adding $G_{l j} \in G S_{l x}$ with $R$ that means $T R^{+}=R+G_{l j}$

II. Compute second augmented representatives $T R^{-}$by adding sign-flipped version of $G_{l j} \in G S_{l x}$ with $R$ that means $T R^{-}=R-G_{l j}$

III. Compute class relevance value $F T_{x}\left(T R^{+}, C\right)$ and $F T_{x}$ $\left(T R^{-}, C\right)$ using $F T_{x}$ score function

IV. If $F T_{x}\left(T R^{+}, C\right) \geq F T_{x}\left(T R^{-}, C\right)$ then If $F T_{x}\left(T R^{+}, C\right)>F T_{x}(R, C)$ then

- Set $R=R+G_{l j}$ and add $G_{l j}$ to $C_{k} G S_{l x}$ and delete $G_{l j}$ from $G S_{l x}$

- $\quad$ count $=$ count +1

V. If $F T_{x}\left(T R^{-}, C\right)>F T_{x}\left(T R^{+}, C\right)$ then If $F T_{x}\left(T R^{-}, C\right)>F T_{x}(R, C)$ then

- Set $R=R-G_{l j}$ and add $G_{l j}$ to $C_{k} G S_{l x}$ and delete $G_{l j}$ from $G S_{l x}$

- $\quad$ count $=$ count +1

g) Set $R=R /$ count

h) Set $A R_{l x k}=R$

E. Select $Q$ number of most relevant cluster representatives according to $F T_{x}$ score from $A R_{l x}$ set and form $R S D_{l x}$ sub data set.

F. Construct a classifier $C_{l x}$ for $R S D_{l x}$ sub data set 
383

384

385

386

387

388

389

390

391

392

393

394

395

396

397

398

399

400

401

402

403

404

405

406

407

408

409

410

411

412

413

414

415

416

417

418

419

420

421

4. Apply a test sample over all the classifiers of all bootstrapped dataset and calculate the prediction accuracy of each classifier

5. Apply simple voting over all predictions to form an ensemble classifier $E C$ and get final prediction.

6. Calculate number of occurrences for every gene for all $R S D_{l x}$ sub datasets across all bootstrapped versions and rank them according to their count.

7. Select a number of top-ranked genes as informative genes.

8. End

\section{Description and Preprocessing of the Datasets}

The experimentation has been carried out over ten publicly available different gene expression binary class and multi-class datasets. Among these datasets, eight datasets are cancer datasets and two arthritis datasets. The eight cancer datasets are Leukemia (Golub1999), Colon (Alon 1999), Prostate (Singh 2002), Lung (Gordon 2002), RBreast (Veer 2002), Breast (West 2001), MLL (Armstrong 2001), and SRBCT (Khan 2001). To show the accuracy of the proposed model with respect to other than cancer datasets here two arthritis datasets RAHC (Pouw Kraan 2003) and RAOA (Pouw Kraan 2007) are also considered. The summary of the datasets is represented in Table 1.

In the Leukemia dataset (Golub1999), the gene expression data matrix is prepared using Affymetrix oligonucleotide arrays. The original dataset consists of two datasets: the training dataset and the testing dataset. The training dataset consists of 38 samples (27 Acute Lymphoblastic Leukemia (ALL) and 11 Acute Myeloid Leukemia (AML)) while the test dataset consists of 34 samples (20 Acute Lymphoblastic Leukemia (ALL) and 14 Acute Myeloid Leukemia (AML)), each with 7129 probes from 6817 genes. For the Leukemia dataset, training and test datasets are merged here and genes with missing values are removed and finally, the dataset with 7070 genes and 72 samples is prepared.

In the Colon cancer dataset (Alon 1999), gene expression of 6500 genes for 62 samples is measured using Affymetrix oligonucleotide arrays. Among these 62 samples, 40 are Colon cancer samples and 22 are normal samples. Among these 6500 genes, 2000 genes are selected based on the confidence of measured expression levels.

Prostate cancer dataset (Singh 2002) also consists of training and testing datasets. In the training dataset, among 102 samples, 50 are normal samples and 52 are prostate cancer samples. In the test dataset among 34 samples, 25 are prostate cancer samples and 9 are normal prostate samples. Gene expression of every sample is measured with respect to 12600 genes using Affymetrix chips. Here, training and test datasets are merged, and a dataset with 12600 genes and 136 samples is formed.

The Lung cancer dataset (Gordon 2002) consists of 181 samples. Among these samples, 31 are malignant pleural mesothelioma and rest150 adenocarcinoma of lung cancer. Each sample is 
422 represented by 12533 genes and the gene expression of every sample is measured using

423 Affymetrix human U95A oligonucleotide probe arrays.

424 In Rbreast data set (Veer 2002), the patients, who are considered as breast cancer patients after 5 425 years intervals of initial diagnosis, fall under the category of relapse and rest as no relapse of 426 metastases. 97 samples have been provided in which 46 patients developed distance metastases

427 within 5 years and they are considered as relapse while the remaining remained healthy and are 428 labeled as non-relapse. This dataset comprises 24481 genes and among them, 293 are removed. 429 In the Breast cancer dataset (West 2001), the gene expression of 49 samples is measured using 430 HuGeneFL Affymetrix microarray arrays. Breast tumors are positive or negative in the presence 431 or absence of estrogen receptors (ER). In this dataset, 25 samples are ER+ tumors and 24 432 samples are ER- tumors.

433 MLL (Armstrong 2001) is a type of dataset which comprises of training data set of 57 leukemia 434 samples including 20 ALL, 17 MLL, and 20 AML and the test dataset including 4 ALL, 3 MLL, 435 and 8 AML samples. For MLL cancer dataset training and test, datasets are merged here and 436 finally, the dataset with 12582 genes and 72 samples are prepared.

437 SRBCT dataset (Khan 2001) is introduced as a dataset comprising of gene-expression for 438 identifying small round blue-cell tumors of childhood SRBCT and samples of this dataset are 439 further divided into four class which are neuroblastoma, rhabdomyosarcoma, non-Hodgkin 440 lymphoma, and Ewing family of tumors and they are obtained from cDNA microarrays. A 441 training set consisting of 63 SRBCT tissues, a test set consisting of 20 SRBCT and 5 non442 SRBCT samples are available. Here we have considered only the training dataset. Each tissue 443 sample is already standardized to zero mean value and has a unit variance across the genes. 444 RAHC commonly known as Rheumatoid Arthritis versus Healthy Controls is a data set (Pouw 445 Kraan 2003) which comprises of gene expression characterizing as peripheral blood cells of 32 446 patients with RA, 3 patients with probable RA, and 15 age with sex-matched healthy controls 447 performed under microarrays with a complexity of 26000 unique genes of 46000 elements. 448 RAOA commonly known as Rheumatoid Arthritis versus Osteoarthritis is a dataset (Pouw Kraan 449 2007) that includes the gene expression of thirty patients in which 21 of them are with RA and 450 the remaining 9 of them are with OA. The Cy5 labeled experimental cDNA and Cy3 labeled 451 common reference sample were pooled and hybridized to the lymphochips (consisting of 18000 452 cDNA spots which symbolize immunology in the genes of relevance).

\section{Results}

454 To assess the performance of the proposed MFSAC-EC model, four well-known existing 455 classifiers named K-Nearest Neighbor (Duda 1999), Naive Bayes (Duda 1999), Support vector 456 machine (Vapnik 1995), and Decision tree(c4.5) (Duda 1999) are applied independently in this 457 model and four different ensemble classification models are formed. To prove the superiority of 458 the proposed model, it is compared with existing well-known filter methods (used here) and 459 existing recognized gene selection methods (Ding 2005, Au2005, Maji2005) and also with 460 different existing ensemble classifiers (Bolo2012, Nagi 2013, Osareh 2013, Wang 2006, Wang 461 2012). To analyze the performance, the methods are applied to different publicly available 
462 cancer and other disease-related gene expression datasets. The major metrics used here for 463 evaluations of the performance of the proposed classifier are the Cross-validation method 464 (LOOCV, 5-fold, and 10-fold), ROC Curve, and Heat map.

\section{Tools Used}

466 The algorithms are implemented using Python programming language and Scikit-learn libraries 467 (Pedregosa 2011) which are explained in (Komer 2014) for ML algorithms. The programs are 468 executed on an online Colab platform with 12 GB RAM and Intel(R) Xeon(R) processor 469 available in the "CPU" Runtime Type at the time of writing. Figures and tables are generated in

470

471

472

473

474

475

476

477

478

479

480

481

482

483

484

485

486

487

488

489

490

491

492

493

494

495

496

497

498

499

500

501 the Matplotlib library (Hunter 2007) and also in Microsoft Excel. The python codes used here are available at https://github.com/NSECResearchCD-SLB/PEERJ MFSAC EC.

In the following subsections, first, the different types of metrics used here are discussed, and then the performance of the proposed MFSAC-EC model is verified with respect to these metrics.

This is followed by comparing the classification performance of the proposed model with different existing methods in terms of 10 -fold cross-validation. The proposed model does not only perform the task of classification but also ranks every attribute or gene in descending order based on its information present in the dataset. To show the effectiveness of this ranking procedure topmost eight genes from Colon cancer and Leukemia cancer datasets are represented with their corresponding names, symbols, and references in significant cancer-related journals to demonstrate their significant roles in these cancers.

\section{Evaluation Metrics}

The performance of the proposed MFSAC-EC classifier is established with respect to the following measures.

\section{Cross-Validation method}

The first well-known metric used here to evaluate the classification model performance is the $k$ fold cross-validation method (Wang 2012). In the $k$-fold cross-validation method, the dataset is randomly divided into $k$ number of folds and $k$ - 1 folds are used for training and one fold is used for testing. The process is repeated for $k$ number of times and average classification accuracy is taken. When $k$ is set at 1 that means the fold size is equal to the size of the dataset (training dataset size is equal to one less than the number of samples in the dataset and validation is done using the remaining sample) then it is considered as Leave one out cross-validation method (LOOCV). For $k$ is equal to 2 , the cross-validation method is named the household method. It has been found that when $k$ is set at a very small value that means the fold size is large then the accuracy of the classification model is affected by low bias and high variance problems. On the other hand, if $k$ is set at a high value that means the fold size is not so large then the classification accuracy of the classification model has a high bias but low variance. It has been found that 10fold cross-validation method outperforms the LOOCV method (Breiman 1992, Ambroise 2002, Asyali 2006) and it has been also endorsed that the 10-fold cross-validation method as a better measure for classification.

In training-testing random splitting the dataset is initially randomly partitioned into training set $\left(2 / 3^{\text {rd }}\right.$ of the dataset) and testing set $\left(1 / 3^{\text {rd }}\right.$ of the dataset $)$ with 50 runs. 


\section{ROC curve analysis}

503 The performance of the proposed classifier for two-class datasets is also judged using Receiver

504 Operator Characteristic (ROC) analysis (Wang 2012). It is a visual method for evaluating binary

505 classification models. Under this analysis, the following measures are considered to judge the

506 binary classification model.

507 Classification accuracy $(A c c)$ is defined as,

508

$$
A C C=\frac{T P+T N}{T P+F P+T N+F N} \quad 0 \leq A C C \leq 1
$$

509 The sensitivity $(S N)$ or True Positive Rate $(T P R)$ can be defined as,

510

$$
S N=T P R=\frac{T P}{T P+F N}
$$

511 The specificity $(S P)$ or True Negative Rate (TNR) can be defined as,

$$
S P=T N R=\frac{T N}{T N+F P}
$$

513 The False Positive Rate $(F P R)$ can be defined as:

$$
F P R=(1-\text { specificity })=\frac{F P}{F P+T N}
$$

515 The Positive Predicted Value $(P P V)$ can be defined as:

516

$$
P P V=\frac{T P}{T P+F P}
$$

The Negative Predicted Value $(N P V)$ can be defined as:

$$
N P V=\frac{T N}{T N+F N}
$$

519

Where TP, TN, FP, FN are true positive, true negative, false positive, and false negative

520 respectively.

521

The ROC curve is plotted considering TPR along the y-axis and FPR along the $\mathrm{x}$-axis. The area

522 under the ROC curve (AUC) is used to represent the performance of the binary classification

523 model. The higher AUC value of a ROC curve for a particular classification model signifies the

524 better performance of the classification model in differentiating positive and negative examples.

525 The range of $\mathrm{AUC}$ value is $0<=\mathrm{AUC}<=1$.

526

527

528

529

530

531

532

533

534

535

536

\section{Heat map analysis}

A heatmap is a data representation diagram in which the values for a variable of interest are portrayed using a data matrix. In this data matrix, the values of the variable are represented across two-axis variables as a grid of colored squares. The axis variables are divided into ranges and each cell's color represents the intensity of that variable for the particular ranges of values of axis variables.

Here, the performance of the proposed classifier for multi-class datasets is judged using Heat map representation of confusion matrix (Liu 2014), where a confusion matrix is a tabular representation to visualize the performance of a classification model in terms of true positive, true negative, false positive and false negative.

\section{Parameter Estimation}

Peer] Comput. Sci. reviewing PDF | (CS-2020:10:54940:1:2:NEW 22 Jun 2021) 
537 Before running the MFSAC-EC, the parameters are settled down. In MFSAC-EC the input 538 training dataset is bootstrapped. The proposed MFSAC-EC model is run here varying the number 539 of bootstrapped datasets $(D)$ from 5 to 30 and the classification accuracy of this model is more or 540 less the same from 10 to the rest of the range. So, the number of bootstrapped datasets for every 541 training dataset for this model is set at 10.

542 In MFSAC method, initially P number of genes is selected by each filter method. Here in Table

543 2, the classification accuracy of the proposed model is shown with respect to different values of

544 P. From Table 2, it has been found that the proposed model gives the best result for $\mathrm{P}=100$ for 545 RAOA and RAHC datasets. In case of Breast cancer, Lung cancer, MLL and SRBCT datasets it

546 gives the best result at $\mathrm{P}=200$. For Leukemia datasets it gives the best result at $\mathrm{P}=500$. So, it 547 can be said that MFSAC-EC gives best result for $\mathrm{P}$ value within 200 to 500 in all cases for all 548 datasets except Colon and Prostate. In Colon and Prostate, it shows the best result for $P=1500$.

549 Here we have used SVM, DT (C4.5), NB, and KNN classifiers individually for forming different 550 ensemble classification models. All the classifiers are implemented using Scilit-learn libraries of 551 Python. For all classifiers, we have set parameters with default parameter values. For DT as 552 default setting we have used splitting function $=$ Gini, Splitting criterion $=$ best, height $=$ none ( 553 that means for every sample it reaches a leaf/class node). For SVM, we have used the RBF 554 kernel function. For KNN we have chosen K (number of nearest neighbor) value from 3 to 7. 555 The overall execution time of a single run of the MFSAC-EC model (considering bootstrapped 556 dataset creation, feature selection using MFSAC, and then generating classification accuracy of 557 test samples using LOOCV, 5-fold, 10-fold, and random splitting) and testing time using only

558

559

560

561

562

563

564

565

566

567

568

569

570

571

572

573

574

575

576 10-fold are shown for different datasets in Table 3.

\section{Classification Performance of the Proposed MFSAC-EC Classifier}

In Table 4, using the LOOCV method, the classification accuracy of our proposed MFSAC-EC model is $100 \%$ for different datasets (Leukemia, Breast, RBreast, Lung, RAOA, and RAHC) for all cases. In the Prostate dataset, we did not get $100 \%$ accuracy using our model with respect to any type of existing classifier. In MLL, Colon, and SRBCT it also gives $100 \%$ accuracy using all types of ensemble classifiers.

In Table 5 and Table 6 , it has been shown that using 5 -fold and 10 -fold cross-validation, MFSAC-EC does not provide $100 \%$ accuracy only for Colon and Prostate cancer datasets. For other datasets, it provides $100 \%$ accuracy with respect to all types of ensemble classifiers. To show the generalization property of the proposed ensemble classifiers, the classification accuracy of these classifiers is also measured repeatedly with respect to the random splitting of the dataset into a training set ( $2 / 3$ data of original dataset) and test set ( $1 / 3$ data of original dataset). Random splitting is done with care such that class proportion is alike in the training set and test set. In Table 7, the classification accuracy of the above mentioned four different types of ensemble classifiers for the different number of cluster representatives is shown in different datasets which are based on the best result of 50 random splitting of the dataset into a training set ( $2 / 3$ data of original dataset) and test set (1/3 data of original dataset). 
577 From the results of Table 4 to Table 7, it has been observed that classification accuracy in the 578 LOOCV method, 5-fold cross-validation, and 10-fold cross-validation methods is higher than the 579 random splitting of the dataset, and the overall generalization performance of the proposed 580 classification model is also good.

581 The performance of the proposed model for different two-class datasets with respect to different 582 parameters like SN, SP, PPV, NPV, FPR is shown in Table 8. From this table, it is found that the 583 performance of the proposed model is very good with respect to all these parameters for all two584 class datasets.

585 In Figures 4, the ROC curve is shown for different two-class datasets. In Figures 4a, 4b, and 4c, 586 the ROC curves are shown for Breast cancer using LOOCV, for Colon cancer using 5-fold cross

587

588

589

590

591

592

593

594

595

596

597

598

599

600

601

602

603

604

605

606

607

608

609

610

611

612

613

614

615 validation, and for RAHC dataset using 10-fold cross-validation respectively. The ROC curves for Leukemia Cancer, and Lung cancer datasets using LOOCV are given in Supplemental Figures F1a and F1b respectively. For Breast cancer, Leukemia cancer, and Lung cancer, the AUC value is equal to 1.0 in every case. The ROC curves are shown for RAOA, and RBreast cancer datasets using 5-fold cross-validation in Supplemental Figures F2a, and F2b respectively. For these datasets also the prediction accuracy using 5-fold cross validation is very high according to the AUC value. In Supplemental Figures F2c, the ROC curves are shown for Prostate cancer using 10-fold cross-validation. From these curves of 10-fold cross validation, it may be seen that except for Prostate cancer, for all other datasets the AUC value is 1 and for Prostate cancer, the AUC value is close to 1.

In Figures $5 \mathrm{a}$ and $5 \mathrm{~b}$, heatmap representation of the confusion matrix are shown for multi-class datasets: SRBCT and MLL with respect to 5-fold cross-validation, and 10-fold cross-validation respectively. From these figures, it is clear that for the proposed model prediction accuracy is accurate in most cases.

\section{Comparison of MFSAC-EC Model with Well-Known Existing Filter Methods used in this model}

In Supplemental Figure F3, the proposed MFSAC-EC model in combination with different existing classifiers is compared with different filter methods used in this model with respect to SRBCT, RAHC, Prostate, and Colon datasets in terms of 10 -fold cross-validation. In all cases, the performance of the proposed model is significantly better with respect to all filters.

\section{Comparison of MFSAC-EC Model with Well-Known Existing Gene Selection Methods}

In Figure 6, the MFSAC-EC model with different existing classifiers as base classifiers are compared with existing well-known supervised gene selection methods named mRMR (minimum redundancy maximum relevance framework) (Ding 2005), MSG (mutual information based supervised gene clustering algorithm) (Maji 2012), CFS (Correlation-based Feature Selection) (Ruiz 2006), and FCBF (Fast Correlation-Based Filter) (Ruiz 2006) with respect to 
616 different classifiers using 10-fold cross-validation method. From these results, it has been found 617 that the proposed model outperforms in most of the cases.

618 In Figure 7, the MFSAC-EC model is compared with well-known existing unsupervised gene 619 selection methods named MGSACO (Tabakhi 2015), UFSACO (Tabakhi 2014), RSM(Lai 620 2006), MC (Haindl 2006), RRFS (Ferreira 2012), TV (Theodoridis 2008), and LS (Liao 2014) 621 with respect to DT, SVM, NB classifiers using random splitting method. From these results, it 622 can be said that the MFSAC-EC model outperforms in all cases.

623

624 Comparison of MFSAC-EC Model with Well-Known Existing Ensemble 625 Classification and DEEP learning Models

626 In Table 9, the proposed MFSAC-EC model using the DT classifier is compared with well627 known existing ensemble classification models with respect to 10-fold cross-validation. These models are PCA-basedRotBoost (Osareh 2013), ICA-based RotBoost (Osareh 2013), AdaBoost

630

631 (Osareh 2013), Bagging (Osareh 2013), Arcing (Osareh 2013), Rotation Forest (Osareh 2013), EN-NEW1 (Wang 2006), and EN-NEW2 (Wang 2006). From Table 9, it is clear that the proposed model using DT classifier outperforms in all cases.

632 In Table 10, the proposed MFSAC-EC model using DT, NB, KNN as base classifiers are compared with different existing ensemble classifiers with respect to 10 -fold cross-validation.

634 These classifiers are Bagging based ensemble classifier (Nagi 2013), Boosting based ensemble

635 classifier (Nagi 2013), Stacking based ensemble classifier (Nagi 2013), Heuristic breadth-first search-based ensemble classifier (HBSA) (Wang 2012), Sd_Ens (Nagi 2013), and Meta_Ens

637 (Nagi 2013). In Table 11 our model using SVM and KNN as base classifiers is compared with

638 auto-encoder-based deep learning models (Nabendu 2020) in terms of random splitting. Here,

639 results are shown only for the datasets for which results are available in the literature, and all

640 other fields are marked as "Not Found". In all cases, the MFSAC-EC model outperforms all the

641 well-known existing ensemble models (except for the Colon cancer dataset) and deep learning

642 models which in turn validates the usefulness of the proposed model.

643

\section{Biological Significance Analysis}

645 The top 8 genes selected by the MFSAC-EC model for Colon cancer and Leukemia are listed in 646 Table 12. For every gene, the name and symbol of the gene as well as the Accession number of 647 the Affymetrix chip are listed. Apart from this information, to validate those genes, biomedical 648 literature of the genes is searched and for every gene, the corresponding reference about its role 649 and significance for a particular disease is provided.

650

\section{1}

\section{Discussion}

652 In this paper, a new Multiple Filtering and Supervised Attribute Clustering algorithm-based 653 ensemble classification model named MFSAC-EC is proposed. The main motivation behind this work is to develop a machine learning-based ensemble classification model to overcome the 655 over-fitting problem which arises due to the presence of sample class imbalance problem, small 
656

657

658

659

660

661

662

663

664

665

666

667

668

669

670

671

672

673

674

675

676

677

678

679

680

681

682

683

684

685

686

687

688

689

690

691

692

693

694

695

sample size problem, and also high dimensional feature set problem in the microarray gene expression dataset, to enhance the prediction capability of the proposed model.

Nowadays, in designing machine learning models, the use of ensemble methodology has been increasing day by day as it incorporates multiple learning algorithms and also training datasets in different efficient manners to improve the overall prediction accuracy of the model. Due to the inclusion of prediction accuracy of multiple learning models and also the use of different bootstrapping datasets, the chances of potential overfitting in training data is greatly reduced in the ensemble models, and as a consequence the prediction accuracy increases. One necessary condition of the superior performance of an ensemble classifier with respect to its individual member/base classifier is that every base classifier should be very accurate and diverse (Osareh 2013). A classifier is considered accurate if its generalization capability is high and two classifiers satisfy diverse property if their prediction in classifying the same unknown samples vary from each other. The general principle of ensemble methods is to rearrange training datasets in different ways (either by resampling or reweighting) and build an ensemble of base classifiers by applying a base classifier on every rearranged training dataset (Osareh 2013). In our proposed ensemble model, at first, a number of bootstrapped datasets of the original training dataset is created. In every bootstrapped dataset, the class imbalance problem is solved using the oversampling method. Then for every bootstrapped dataset, a number of sub-datasets are created using the MFSAC method (which is a hybrid method combining multi-filters and a new supervised attribute/gene clustering method), and then for every generated sub dataset, a base classifier is constructed using any existing classification model. After that, a new ensemble classifier (EC) is formed using the majority voting scheme by combining the prediction accuracy of all those base classifiers.

The prediction accuracy of the proposed model is verified by applying it to high-dimensional microarray gene expression data From Figure 6, and Figure 7 it has been found that the classification accuracy of the MFSAC-EC model is much better than the well-known existing gene selection methods. From Table 9, Table 10, and Table 11, it has been also found that the proposed MFSAC-EC classification model is superior to the existing ensemble classification models in almost every case. The superior performance of the proposed model is due to the following reasons:

- The generation of the different bootstrapped versions of training data and also the use of the oversampling procedure to balance the cardinality of majority class and minority class in every bootstrapped dataset reduces the chances of the overfitting problem of a classifier.

- Different types of filter methods are used in the MFSAC method. It has been already observed that one filter gives better performance for one dataset while the same gives poor results for other datasets. This is because every filter uses separate metrics and so the choice for a filter for a specific dataset is a very complex task. As different filter methods are used in the MFSAC method, so different sub-datasets with different characteristics-based attributes/genes are formed from each dataset. This is shown using 
696

697

698

699

700

701

702

703

704

705

706

707

708

709

710

711

712

713

714

715

716

717

718

719

720

721

722

723

724

725

726

727

728

729

730

731

732

733

734

735

Venn diagram in Supplemental Figures F4a and F4b. Here for Leukemia and Prostate cancer datasets, the first twenty genes, selected by each filter are shown. In case of Leukemia dataset, Relief measure generates non-overlapping gene subset while using other filter metrics presence of a small number of overlapping genes in different gene subsets are observed. In Prostate cancer dataset, Relief generates non-overlapping gene subset and also maximum number of genes are non-overlapping in gene subsets formed by Fisher score, MI (mutual information). From these figures, it is clear that using different filter methods different subsets of genes are selected and different sub datasets are formed. It shows diversity of those filter methods. As a consequence, the base classifiers prepared on these diverse datasets are become diverse. This diversity increases the power of ensemble classifier.

- Moreover, the genes selected by different filter methos are good biomarker also. In Table 12, the top ranked 8 genes selected by MFSAC-EC model are shown for Leukemia and Colon cancer datasets. Among these genes, gene MPO (with column number 1720), CST3 (with column number 1823), ZYX (with column number 4788), CTSD (with column number 2062), CD79A/MB-1(with column number 2583), LYZ (with column number 6738) in Leukemia dataset are important biomarkers as these are selected by different filter methods mentioned in Supplemental Figure F4.

- In MFSAC, at first, a sub dataset of the most relevant genes is selected by each filter method. Then on each sub dataset, the proposed supervised gene clustering algorithm is applied and a reduced sub dataset of modified attributes/features in the form of augmented cluster representatives is generated. In this method, at the time of cluster formation, genes are augmented based on their supervised information. In other words, such augmentation is considered where it increases the class discrimination power. Thus effectively, the class relevance of any augmented cluster representative is greater than that of any single gene involved in that process. So, this modified sub dataset containing a reduced feature set in the form of augmented cluster representatives is more powerful according to class discrimination power than the sub dataset containing a subset of the most relevant genes. Apart from this, it is well known fact in gene expression data that two genes are functionally similar if they are pattern-based similar (either positively coexpressed or negatively co-expressed) (Das 2016). So, at the time of the augmentation procedure, two types of augmentations are considered here. One is that a gene is added with its original value with the current cluster representative and another one is that the gene is added with its sign-flipped value with the current cluster representative. This is because if the current cluster representative and a gene are positively co-expressed then normal addition is considered but if they are negatively co-expressed then normal addition will hamper the addition process and in that case, sign-flipping of that gene will give proper result. The effect of augmentation with respect to every filter method is shown in Figure 8. In Figure 8, for the Breast cancer dataset, at the time of supervised cluster formation from each filter generated subset, the original gene, and its

Peer] Comput. Sci. reviewing PDF | (CS-2020:10:54940:1:2:NEW 22 Jun 2021) 
736

737

738

739

740

741

742

743

744

745

746

747

748

749

750

751

752

753

754

755

756

757

\section{8}

759

760

761

762

763

764

765

766

767

768

769

770

771

772

773

774

775

corresponding class relevance value, and also augmented gene and its corresponding class relevance are shown. From Figure 8, it is clear that for every filter method the class relevance score of every original gene is increased with respect to that filter after augmentation. In Figure 8, different class labels are distinguished by different colors.

- Finally, for each sub dataset with modified attributes in the form of augmented cluster representatives, a classifier is constructed using any existing classifier, and these classifiers are combined using the majority voting technique to form an ensemble classifier (EC). The use of different sub-datasets with optimal gene subsets in the form of augmented cluster representatives and the formation of a classifier for every sub dataset can solve the overfitting problem of any single classifier. This is due to the reason that not all sub-datasets can consistently perform well on all types of cancer datasets (due to inherent characteristics of the datasets), but due to the use of majority voting in ensemble classifiers, this problem can be solved or reduced.

Another outcome of our proposed model is to rank informative genes for every cancer dataset.

For this task, the frequency of occurrence of each gene present in the form of augmented cluster representatives in every sub dataset is counted and these genes are ranked according to the counted value to measure the importance of those genes for any specific disease, here cancer. To establish the biological significance of those selected genes for every cancer dataset, their contribution has been confirmed by other existing studies where they are referred already. From these existing studies, it is clear that the selected genes are important for cancer class discrimination and also are important as cancer biomarkers for molecular treatment targets.

\section{Conclusions}

Many machine learning and statistical learning-based classifiers for sample classification already exist in the literature, but these methods are prone to suffer from overfitting due to small sample size problems, class imbalance problems, and the curse of the high dimensionality of microarray data. Although some of the existing methods can mitigate these issues to quite an extent, the problems have still not been satisfactorily overcome. Due to this reason, here a novel feature selection-based ensemble classification model named MFSAC-EC is proposed. It has been shown that the proposed model can handle the above-mentioned issues present in existing models. To check the performance of the proposed MFSAC-EC model, this classifier is applied to test sample classification accuracy in high dimensional microarray gene expression data, a domain that will be beneficial in the field of cancer research. From the experimental results, it has been found that the proposed model outperforms all other well-known existing classification models combined with the different recognized feature selection methods and also the newly developed ensemble classifiers for all types of cancer datasets mentioned here. Apart from this classification task, the proposed model can also rank informative attributes according to their importance. The efficiency of the proposed model in this task is vindicated by finding the most informative genes for Colon cancer and Leukemia cancer datasets using this model. These genes are biologically validated based on other well-known existing studies. Consequently, it is clear 
776 that the selected genes are vital for sample class discrimination and are also important

777 biomarkers for molecular treatment targets of deadly diseases.

778

779

Acknowledgments

780

781

782

783

784

785

786

787

788

789

790

791

792

793

794

795

796

797

798

799

800

801

802

803

804

\section{References}

Alon U, Barkai N, Notterman D. A., Gish K., Ybarra S., Mack D., and Levine A. J. 1999. Broad patterns of gene expression revealed by clustering analysis of tumor and normal colon tissues probed by Oligonucleotide arrays. Proceedings of National Academy of Sciences, USA. 96(12): 6745-6750.

Ambroise C, McLachlan GJ. 2002. Selection bias in gene extraction on the basis of microarray gene-expression data. Proceedings of National Academy of Sciences, USA. 99(10):6562-6566.

Armstrong S, Staunton J, Silverman L, Pieters R, den Boer M, Minden M, Sallan S, Lander E, Golub T and Korsmeyer S. 2001. MLL translocations specify a distinct gene expression profile that distinguishes a unique leukemia. Nature Genetics. 30:41-47.

Asyali MH, Colak D, Demirkaya O, Inan MS. 2006. Gene expression profile classification: A review. Current Bioinformatics. 1(1):55-73.

Austin H Chen, Yin-Wu Tsau and Ching-Heng Lin. 2010. Novel methods to identify biologically relevant genes for leukemia and prostate cancer from gene expression profiles. BMC Genomics.

Au W. H., Chan K. C. C., Wong A.K.C., and Wang Y. 2005. Attribute clustering for grouping, selection, classification of gene expression data. IEEE/ACM Transactions of Computational Biology and Bioinformatics.

2(2):83-101.

Bai YX, Yi Ji-Lin, Li Jian-Feng, Hong Sui. 2007 Clinicopathologic significance of BAG1 and TIMP3 expression in colon carcinoma. World Journal of Gastroenterology. 13(28): 3883-3885.

Błaszczyński Jerzy, Stefanowskił Jerzy, Idkowiak ukasz.2013. Extending Bagging for Imbalanced Data.

Proceedings of the 8th International Conference on Computer Recognition Systems (CORES 2013).

Bolo' n-Canedo V, Sánchez-Maroño Noelia, Alonso-Betanzos Amparo. 2012. An ensemble of filters and classifiers for microarray data classification. Pattern Recognition. 45(1):531-539.

Botchkina Inna L, Roweh Rebecca A, Rivadeneira David E, Karpeh Martin S Jr, Crawford Howard, Dufour

Antoine, Ju Jingfang, Wang Yuan, Leyfman Yan, Botchkina Galina I. 2009. Phenotypic Subpopulations of

Metastatic Colon Cancer Stem Cells: Genomic Analysis. Cancer Genomics \& Proteomics. 6(1): 19-30.

807

Breiman L, Spector P. 1992. Submodel selection and evaluation regression - the X-random case. International

808

809

810

811

812

813 Statistical Review. 60(3):291-319.

Brown Adam R, Simmen C.M. Rosalia, Raj Vinay R., Van Trang T., MacLeod Stewart L, Simmen Frank A. 2015. Krüppel-like factor 9 (KLF9) prevents colorectal cancer through inhibition of interferon-related signaling. Carcinogenesis. 36(9): 946-955.

Chen Xiao, Gao Bing, Ponnusamy Murugavel, Lin Zhijuan, Liu Jia. 2017. MEF2 signaling and human diseases. Oncotarget. 8(67):112152-112165.

814 Chin A J, Mirzal A, Haron H, Hamed H N A. 2016. Supervised, Unsupervised and Semi-supervised Feature

815 Selection: A Review on Gene Selection. IEEE Transactions on Computational Biology and Bioinformatics.

816 13(5):971-989.

817 Colozza M, Cardoso F, Sotiriou C, Larismont D, Piccart M J. 2005. Bringing molecular prognosis and prediction to

818 the clinic. Clin Breast Cancer. 6(1):61-76.

819 Das Chandra, Bose Shilpi, Chattopadhyay Matangini, Chattopadhyay Samiran. 2016. A novel distance-based

820 iterative sequential KNN algorithm for estimation of missing values in microarray gene expression data.

821 IJBRA.12(4):312.

822 Das Chandra, Bose Shilpi, Banerjee Abhik, Dutta Sourav, Ghosh Kuntal, Chattopadhyay Matangini. 2019.

823 Comparative Performance Analysis of Different Measures to Select Disease Related Informative Genes from

Peer] Comput. Sci. reviewing PDF | (CS-2020:10:54940:1:2:NEW 22 Jun 2021) 
824 Microarray Gene Expression Data. International Conference on Innovation in Modern Science and Technology

825 (ICIMSAT-2019), Springer.

826 Dashtban M. and Balafar M. 2017. Gene selection for microarray cancer classification using a new evolutionary

827

828

829

830

831

832

833

834

835

836 method employing artificial intelligence concepts. Genomics.109(2): 91-107.

Dettling M and Buhlmann P. 2002. Supervised Clustering of Genes. Genome Biology. 3(12): 0069.1-0069.15.

Dietterich T. G.. 2000. An experimental comparison of three methods for constructing ensembles of decision trees: bagging, boosting, and randomization. Machine Learning. 40(2): 139-157.

Ding C. and Peng H. 2005. Minimum redundancy feature selection from microarray gene expression data. Journal of Bioinformatics and Computational Biology. 3(2): 185-205.

Duda R. O., Hart P. E., and Stork D. G.1999. Pattern Classification and Scene Analysis. NewYork:Wiley.

Durai Rajaraman, Yang Y Shi, Seifalian M Alexander, Goldspink Geoffrey, Winslet C Marc. 2007. Role of

insulin-like growth factor binding protein-4 in prevention of colon cancer. World Journal of Surgical Oncology. 5:128.

837 Elyasigomari V, Lee D. A., Screen H. R.C, Shaheed M.H. 2017. Development of a two-stage gene selection method that incorporates a novel hybrid approach using the cuckoo optimization algorithm and harmony search for cancer classification. Journal of Biomedical Informatics. 67:11-20.

840 Feng Hailiang, Liu Yanyan, Bian Xiaocui, Zhou Fangying, Liu Yuqin. 2018. ALDH1A3 affects colon cancer in vitro proliferation and invasion depending on CXCR4 status. British Journal of Cancer. 118: 224-232.

842 Ferreira A.J., Figueiredo M.A.T. 2012. An unsupervised approach to feature discretization and selection, Pattern

843 Recognition. 45: 3048-3060.

844 Furey T.S., Cristianini N, Duffy N, Bednarski D.W, Schummer M, and Haussler D. 2000. Support Vector Machine

845 Classification and Validation of Cancer Tissue Samples Using Microarray Expression Data. Bioinformatics. 16(10):

846 906-914.

847 Gardina Paul J, Clark Tyson A, Shimada Brian, Staples Michelle K, Yang Qing, Veitch James, Schweitzer Anthony, 848 Awad Tarif, Sugnet Charles, Dee Suzanne, Davies Christopher, Williams Alan, Turpaz Yaron. 2006. Alternative 849 splicing and differential gene expression in colon cancer detected by a whole genome exon array. BMC

850 GENOMICS. 7: 325

851 Ghorai Santanu, Mukherjee Anirban, Sengupta Sanghamitra, Dutta Pranab K.. 2011. Cancer Classification from

852 Gene Expression Data by NPPC Ensemble. IEEE/ACM Transactions on Computational Biology and

853 Bioinformatics. 8(3).

854 Giorgio Eros Di, Hancock Wayne W, Brancolini Claudio. 2018. MEF2 and the tumorigenic process, hic sunt leones.

855 BBA - Reviews on Cancer. 1870(2):261-273.

856 Golub T. R., Slonim D. K., Tamayo P, Huard C, Gaasenbeek M, Mesirov J.P., Coller H, Loh H.L., Downing J. R.,

857 Caligiuri M. A., Bloomfield C. D., and Lander E. S.. 1999. Molecular Classification of Cancer: Class Discovery and 858 Class Prediction by Gene Expression Monitoring. Science. 286(5439):531-537.

859 Gordon G. J., Jensen R. V., Hsiao L.-L., Gullans S. R., Blumenstock J. E., Ramaswamy S., Richards W. G.,

860 Sugarbaker D. J., and Bueno R. 2002. Translation of microarray data into clinically relevant cancer diagnostic tests 861 using gene expression ratios in lung cancer and mesothelioma. Cancer Research. 62: 4963-4967.

862 Greller LD, Tobin FL. 1999. Detecting selective expression of genes and proteins. Genome Research. 9:282-296.

863 Gu Q., Li Z., Han J.. Generalized Fisher Score for Feature Selection. 2011. UAI'11: Proceedings of the Twenty-

864 Seventh Conference on Uncertainty in Artificial Intelligence.

865 Hai Wang, Hu Haiyan, Zhang Qian, Yang Yadong, Li Yanming, Hu Yang, Ruan Xiuyan, Yang Yaran, Zhang

866 Zhaojun, Shu Chang, Yan Jiangwei, Wakeland Edward K, Li Quanzhen, Hu Songnian, Fang Xiangdong. 2013.

867 Dynamic transcriptomes of human myeloid leukemia cells. Genomics. 102:250-256.

868 Haindl M, Somol P, Ververidis D, Kotropoulos C.2006. Feature Selection Based on Mutual Correlation. Pattern 869 Recognition, Image Analysis and Applications. Springer Berlin Heidelberg. 569-577.

870 Hambali Moshood A., Oladele Tinuke O., Adewole Kayode S. 2020. Microarray cancer feature selection: Review, 871 challenges and research directions. International Journal of Cognitive Computing in Engineering.1: 78-97. 
872 Handschuh Luiza. 2019. Not Only Mutations Matter: Molecular Picture of Acute Myeloid Leukemia Emerging from

873 Transcriptome Studies. Journal of Oncology.

874 Hastie T, Tibshirani R, Eisen M.B., Alizadeh A, Levy R, Staudt L, Chan W. C., Botstein D, and Brown P. 2000.

875 'Gene Shaving' as a Method for Identifying Distinct Sets of Genes with Similar Expression Patterns. Genome

876 Biology. 1(2):1-21.

877 Hastie T, Tibshirani R, Botstein D, and Brown P. 2001. Supervised Harvesting of Expression Trees. Genome

878 Biology. 1: 1-12.

879 Hunter JD. 2007. Matplotlib: a 2D graphics environment. Computing In Science \& Engineering 9(3):90-95 DOI

880 10.1109/MCSE.2007.

881 Kamal Amany M, El-Hefny Nadia H, Hegab Hany M, El-Mesallamy Hala O. 2016. Expression of thioredoxin-1

882 (TXN) and its relation with oxidative DNA damage and treatment outcome in adult AML and ALL: A comparative

883 study. Hematology. 21(10):567-575.

884 Karlenius Therese Christina, Tonissen Kathryn Fay. 2010. Thioredoxin and Cancer: A Role for Thioredoxin in all

885 States of Tumor Oxygenation. Cancers (Basel). 2(2): 209-232.

886 Kim Yundeok, Yoon Sulhee, Kim Soo Jeong, Kim Jin Seok, Cheong Jun-Won, Hong Yoo Min. 2012.

887 Myeloperoxidase Expression in Acute Myeloid Leukemia Helps Identifying Patients to Benefit from Transplant.

888 Yonsei Med J. 53(3): 530-536.

889 Khan J, Wei JS, Ringner M, Saal LH, Ladanyi M, Westermann F, Berthold F, Schwab M, Antonescu CR, Peterson

890 C, Meltzer S Paul. 2001. Classification and diagnostic prediction of cancers using gene expression profiling and

891 artificial neural networks. Nat Medicine. 7(6):673-679.

892 Klimiankou Maksim, Uenalan Murat, Kandabarau Siarhei, Nustede Rainer, Steiert Ingeborg, Mellor-Heineke

893 Sabine, Zeidler Cornelia, Skokowa Julia, Welte Karl. 2019. Ultra-Sensitive CSF3R Deep Sequencing in Patients

894 With Severe Congenital Neutropenia. Front. Immunol.

895 Komer B, Bergstra J, Eliasmith C. 2014. Hyperopt-sklearn: automatic hyperparameter configuration for scikit-learn.

896 In: Proceedings of the 13th Python in Science Conference (SCIPY 2014). 33-39.

897 Kourou Konstantina, Exarcos T P, Exarcos K P, Karmouzis M V, Fotaidis D. 2015. Machine learning applications

898 in cancer prognosis and prediction. Computational and Structural Biotechnology Journal. 13: 8-17.

899 Kozlov Igor, Beason Kevin, Yu Cheng, Hughson Michael. 2005, CD79a expression in acute myeloid leukemia

$900 \mathrm{t}(8 ; 21)$ and the importance of cytogenetics in the diagnosis of leukemias with immunophenotypic ambiguity. Cancer

901 Genetics and Cytogenetics 163(1):62-7.

902 Kozovska. Z, Patsalias A., Bajzik V, Durinikova E, Demkova L, Jargasova S, Smolkova B, Plava J, Kucerova L,

903 Matuskova M. 2018. ALDH1A inhibition sensitizes colon cancer cells to chemotherapy. BMC Cancer. 18:656.

904 Lagunas-Rangel Francisco Alejandro, Chávez-Valencia Venice, Gómez-Guijosa Miguel Ángel, Cortes-Penagos

905 Carlos. 2017. Acute Myeloid Leukemia - Genetic Alterations and Their Clinical Prognosis. Int J Hematol Oncol

906 Stem Cell Res. 11(4): 328-339.

907 Lai C, Reinders MJT, Wessels L. 2006. Random subspace method for multivariate feature selection. Pattern

908 Recognition Letters., 27: 1067-1076.

909 Lance Amanda, Druhan Lawrence J, Vestal C Greer, Steuerwald Nury M, Hamilton Alicia, Smith Mathew, Price

910 Andrea, Tjaden Elise, Fox Andee N, Avalos Belinda R. 2020. Altered expression of CSF3R splice variants impacts

911 signal response and is associated with SRSF2 mutations. Leukemia. 34(2):369-379.

912 Leung Yukyee and Hung Yeungsam. 2010. A Multiple-Filter-Multiple-Wrapper Approach to Gene Selection and

913 Microarray Data Classification”, IEEE/ACM Transactions on Computational Biology and Bioinformatics. 7(1).

914 Léveillard Thierry, Aït-Ali Najatel. 2017. Cell Signaling with Extracellular Thioredoxin and Thioredoxin-Like

915 Proteins: Insight into Their Mechanisms of Action. Oxidative Medicine and Cellular Longivity.

916 Li Z, Xie W, Liu T. 2018. Efficient feature selection and classification for microarray data. Plos one.

917 Liao B, Jiang Y, Liang W, Zhu W, Cai L, Cao Z. 2014. Gene selection using locality sensitive Laplacian score.

918 IEEE/ACM Transactions on Computational Biology and Bioinformatics. DOI: 10.1109/TCBB.2014.2328334.

919 Liu Jian, Cheng Yuhu, Wang Xuesong, Zhang Lin, Wang Z. Jane. 2018. Cancer Characteristic Gene Selection via

920 Sample Learning Based on Deep Sparse Filtering. Scientific Reports, Nature. 8: 8270.

Peer] Comput. Sci. reviewing PDF | (CS-2020:10:54940:1:2:NEW 22 Jun 2021) 
921 Liu Q, Sung A H, Chen Z, Liu J, Chen L, Qiao M, Wang Z, Huang X, Deng Y. 2011. Gene selection and

922 classification for cancer microarray data based on machine learning and similarity measures. BMC Genomics.

923 12(5):S1.

924 Liu Sheng, Dissanayake Shamitha, Patel Sanjay V, Dang Xin. 2014. Learning accurate and interpretable models

925 based on regularized random forests regression. BMC Systems Biology. 8 Suppl 3(Suppl 3):S5.

926 M Lizet. Waals van der, M. H. Inne. Rinkes Borel, Kranenburg Onno. 2018. ALDH1A1 expression is associated

927 with poor differentiation, 'right-sidedness' and poor survival in human colorectal cancer. PLOS ONE.

928 Maji P, Das C. 2012. Relevant and Significant Supervised Gene Clusters for Microarray Cancer Classification. IEEE

929 Transactions on Nanobioscience. 11(2).

930 Nabendu Bhui, Pintu Kumar Ram, Pratyay Kuila. 2020. Feature Selection from Microarray Data based on Deep

931 Learning Approach. ICCCNT2020.

932 Nada A, Alshamlan H. 2019. A survey on hybrid feature selection methods in microarray gene expression data for

933 cancer classification", IEEE Access.

934 Nagi Sajid, Bhattacharyya Kr. Dhruba. 2013. Classification of microarray cancer data using ensemble approach.

935 Netw Model Anal Health Inform Bioinformatics. 2:159-173.

936 Osareh Alireza, Bita Shadgar. 2013. An Efficient Ensemble Learning Method for Gene Microarray Classification.

937 BioMed Research International, Hindawi Publishing Corporation.

938 Pedregosa F, Varoquaux G, Gramfort A, Michel V, Thirion B, Grisel O, Blondel M, Prettenhofer P, Weiss R,

939 Dubourg V, Vanderplas J, Passos A, Cournapeau D, Brucher M, Perrot M, Duchesnay E. 2011. Scikit-learn:

940 machine learning in python. Journal of MachineLearning Research 12:2825-2830.

941 Pilling M J, Henderson A, Gardner P. 2017. Quantum Cascade Laser Spectral Histopathology: Breast Cancer

942 Diagnostics Using High Throughput Chemical Imaging. Analytical Chemistry. 89 (14), 7348-7355.

943 Pouw Kraan T.C.T.M. Van der, Gaalen F.A. van, Kasperkovitz P.V., Verbeet N.L., Smeets T.J.M., Kraan M.C.,

944 Fero M., Tak P.-P., Huizinga T.W.J., Pieterman E., Breedveld F.C., Alizadeh A.A., , and Verweij C.L. 2003.

945 Rheumatoid Arthritis is a Heterogeneous Disease:Evidence for Differences in the Activation of the STAT-1

946 Pathway between Rheumatoid Tissues. Arthritis and Rheumatism. 48(8):2132-2145.

947 Pouw Kraan T.C.T.M. van der, Wijbrandts C.A., Baarsen L.G.M. van, Voskuyl A.E., Rustenburg F., Baggen J.M.,

948 Ibrahim S.M., Fero M., Dijkmans B.A.C., Tak P.P., and Verweij C.L. 2007. Rheumatoid Arthritis Subtypes

949 Identified by Genomic Profiling of Peripheral Blood Cells: Assignment of a Type I Interferon Signature in a

950 Subpopulation of Pateints. Annals of the Rheumatic Diseases. 66: 1008-1014.

951 Ritter Malte, Klimiankou Maksim, Klimenkova Olga, Schambach Axel, Hoffmann Dirk, Schmidt Amy, Kanz

952 Lothar, Link Daniel C., Welte Karl, Skokowa Julia. 2020. Cooperating, congenital neutropenia-associated Csf3r and

953 Runx1 mutations activate pro-inflammatory signaling and inhibit myeloid differentiation of mouse HSPCs. Annals

954 of Hematology. 99: 2329-2338.

955 Ruiz R., Riquelme J.C., Aguilar-Ruiz J.S. 2006. Incremental wrapper-based gene selection from microarray data for

956 cancer classification. Journal of Pattern Recognition. 39(12): 2383-2392.

957 Simmen Frank A, Su Ying, Xiao Rijin, Zeng Zhaoyang, Simmen CM Rosalia. 2008. The Krüppel-like factor 9

958 (KLF9) network in HEC-1-A endometrial carcinoma cells suggests the carcinogenic potential of dys-regulated

959 KLF9 expression. Reproductive Biology and Endocrinology.

960 Singh D., Febbo P.G., Ross K., Jackson D. G., Manola J., Ladd C., Tamayo P., Renshaw A. A., Amico A. V. D,

961 Richie J. P., Lander E. S., Loda M., Kantoff P.W., Golub T.R., and Sellers W.R..2002. Gene expression correlates

962 of clinical prostate cancer behavior. Cancer Research..1: 203-209.

963 Singh P, Dai B, Dhruva B, Widen S G 1994. Episomal Expression of Sense and Antisense Insulin-like Growth

964 Factor (IGF) binding Protein-4 Complementary DNA Alters the Mitogenic Response of a Human Colon Cancer Cell

965 Line (HT-29) by Mechanisms That Are Independent of and Dependent upon IGF-11. Cancer Research. 54: 6563-

9666570.

967 Su AI, Welsh JB, Sapinoso LM, Kern SG, Dimitrov P, Lapp H, Schultz PG, Powell SM, Moskaluk CA, Frierson

968 HF, Hampton GM. 2001. Molecular classification of human carcinomas by use of gene expression signatures.

969 Cancer Research. 61:7388-7393.

Peer] Comput. Sci. reviewing PDF | (CS-2020:10:54940:1:2:NEW 22 Jun 2021) 
970

971

972

973

974

975

976

977

978

979

980

981

982

983

984

985

986

987

988

989

990

991

992

993

994

995

996

997

998

999

1000

1001

1002

1003

1004

1005

1006

1007

1008

1009

1010

1011

1012

1013

1014

1015

1016

1017

1018

Su Chun-Wen, Lin Chiao-Wen, Yang Wei-En, Yang Shun-Fa. 2019. TIMP-3 as a therapeutic target for cancer. Therapeutic Advances in Medical Oncology.

Su Li, Luo Yongli, Yang Zhi, Yang Jing, Yao Chao, Cheng Feifei, Shan Juanjuan, Chen Jun, Li Fangfang, Liu Limei, Liu Chungang, Xu Yanmin, Jiang Lupin, Deyu Guo, Prieto Jesus, Ávila A Matías, Shen Junjie, Qian Cheng. 2016. MEF2D Transduces Microenvironment Stimuli to ZEB1 to Promote Epithelial-Mesenchymal Transition and Metastasis in Colorectal Cancer. Molecular and Cellular Pathobiology. 76(17):5054-5067.

Swan A L, Mobasheri Ali, Allaway David, Liddell Susan, Bacardit Jaume. 2013. Application of Machine Learning to Proteomics Data: Classification and Biomarker Identification in Postgenomics Biology. OMICS. 17(12): 595610.

Szuber Natasha, Tefferi Ayalew. 2018. Chronic neutrophilic leukemia: new science and new diagnostic criteria. Blood Cancer Journal. 19.

Tabares-Soto Reinel, Orozco-Arias Simon, Romero-Cano Victor, Segovia Bucheli Vanesa, Rodríguez-Sotelo José Luis , Jiménez-Varón Cristian Felipe. 2020. A comparative study of machine learning and deep learning algorithms to classify cancer types based on microarray gene expression data. PeerJ Computer Science.

Tabakhi S, Moradi P, Akhlaghian F. 2014. An unsupervised feature selection algorithm based on ant colony optimization. Engineering Applications of Artificial Intelligence. 32: 112-123.

Tabakhi S, Najafi, A., Ranjbar, R., \&Moradi, P. 2015. Gene selection for microarray data classification using a novel ant colony optimization. Neurocomputing, 168: 1024-1036.

Tang Zhenjie, Yuan Shuqiang, Hu Yumin, Zhang Hui, Wu Wenjing, Zeng Zhaolei, Yang Jing, Yun Jingping, Xu Ruihua, Huang Peng. 2012. Over-expression of GAPDH in human colorectal carcinoma as a preferred target of 3Bromopyruvate Propyl Ester" Published in final edited form as: J Bioenerg Biomembr. 44(1): 117-125.

Theodoridis S, Koutroumbas K. 2008. Pattern Recognition, fourth ed., Elsevier Science. Thorsen Kasper, Sørensen D Karina, Brems-Eskildsen Sofie Anne, Modin Charlotte, Gaustadnes Mette, Hein K Anne-Mette, Kruhøffer Mogens, Laurberg Soren, Borre Michael, Wang Kai, Brunak Søren, Krainer R Adrian, Tørring Niels , Dyrskjøt Lars, Andersen Claus L, Orntoftet Torben F.2008. Alternative Splicing in Colon, Bladder, and Prostate Cancer Identified by Exon Array Analysis. Molecular \& Cellular Proteomics. 7: 1214-1224.

Tong Dong Ling, Ball Graham R. 2014. Exploration of Leukemia Gene Regulatory Networks Using A Systems Biology Approach. 2014 IEEE International Conference on Bioinformatics and Biomedicine.

Vapnik V. 1995. The Nature of Statistical Learning Theory. New York: Springer-Verlag. Veer L. J. V., Dai H., Vijver M. J. V. D., He Y. D., Hart A. A.M., Mao M., Peterse H. L., Kooy K. v. d., Marton M. J., Witteveen A. T., Schreiber G.J. , Kerkhoven R.M., Roberts C. , Linsley P. S., Bernards R., and Friend S. H..2002. Gene expression profiling predicts clinical outcome of breast cancer. Nature. 415(6871): 530-536.

Wang Ching Wei. 2006. New Ensemble Machine Learning Method for Classification and Prediction on Gene Expression Data. Proceedings of the 28th IEEE EMBS Annual International Conference New York City, USA. Wang Shu-Lin, Li Xue-Ling, Fanget Jianwen. 2012. Finding minimum gene subsets with heuristic breadth-first search algorithm for robust tumor classification. BMC Bioinformatics.13:178.

West M., Blanchette C., Dressman H., Huang E., Ishida S., Spang R., Zuzan H., Olson J. A., Marks J. R., and Nevins J. R.. 2001. Predicting the clinical status of human breast cancer by using gene expression profiles. Proc. Natl. Acad. Sci. USA. 98(20):11 462-11 467.

Yang P, Zhou B Bing, Zhang Zili, Zomaya Y, Albert. 2010. A multi-filter enhanced genetic ensemble system for gene selection and sample classification of microarray data. BMC Bioinformatics. 11: S5.

Yan Zhi, Li Jiangeng, Xiong Yimin, Xu Weitian, Zheng Guorong. 2012. Identification of candidate colon cancer biomarkers by applying a random forest approach on microarray data. Oncology Reports, SPANDIDOS Publications. 28(3):1036-42.

Yunsong Q, Yang Xibei. 2013. Interval-valued analysis for discriminative gene selection and tissue sample classification using microarray data. Genomics. 101(1):38-48.

Ying Mingyao, Tilghman Jessica, Wei Yingying, Guerrero-Cazares Hugo, Alfredo Quinones-Hinojosa, Ji Hongkai, Laterra John. 2014. KLF9 Inhibits Glioblastoma Stemness through Global Transcription Repression and Integrin- $\alpha 6$ Inhibition. Journal for Biochemistry and Molecular Biology. 289(47): 32742-32756.

Peer] Comput. Sci. reviewing PDF | (CS-2020:10:54940:1:2:NEW 22 Jun 2021) 
1019 Yu Herbert, Rohan Thomas. 2000. Role of the Insulin-Like Growth Factor Family in Cancer Development and 1020 Progression. Journal of the National Cancer Institute. 92(18): 1472-1489.

1021 Zhang Jin-Ying, Zhang Fan, Hong Chao-Qun, Giuliano Armando E., Cui Xiao-Jiang, Zhou Guang-Ji, Zhang Guo1022 Jun, Cui Yu-Kun. 2015. Critical protein GAPDH and its regulatory mechanisms in cancer cells. Cancer Biol Med 1023 12(1):10-22.

1024 Zhang Yang, Wang Fang, Chen Xue, Zhang Yu, Wang Mingyu, Liu Hong, Cao Panxiang, Ma Xiaoli, Wang Tong, 1025 Zhang Jianping, Zhang Xian, Lu Peihua, Liu Hongxing 2018. CSF3R Mutations are frequently associated with 1026 abnormalities of RUNX1, CBFB, CEBPA, and NPM1 genes in acute myeloid leukemia. Cancer. 124(16):332910273338.

1028 Zhou Nina and Wang Lipo. 2007. A Modified T-test Feature Selection Method and Its Application on the HapMap 1029 Genotype Data. Genomics Proteomics Bioinformatics. 5(3-4): 242-9.

1030 Zhu Kongxi, Wang Yunxia, Liu Lan, Li Shuai, Yu Weihua. 2019. Long non-coding RNA MBNL1-AS1 regulates 1031 proliferation, migration, and invasion of cancer stem cells in colon cancer by interacting with MYL9 via sponging 1032 microRNA-412-3p. Clinics and Research in Hepatology and Gastroenterology. 44(1):101-114. 


\section{Table $\mathbf{1}$ (on next page)}

Description of Cancer Gene Expression Datasets 
Table 1. Description of Cancer Gene Expression Datasets

\begin{tabular}{|c|c|c|c|c|c|c|c|}
\hline Dataset & $\begin{array}{c}\text { Data Dimension } \\
\text { Gene } \times \text { Sample } \\
(\text { Original })\end{array}$ & $\begin{array}{c}\text { Data Dimension } \\
\text { Gene } \times \text { Sample } \\
(\text { Used })\end{array}$ & $\begin{array}{c}\text { Sample } \\
\text { Class } \\
\text { Labels }\end{array}$ & Dataset & $\begin{array}{c}\text { Data Dimension } \\
\text { Gene } \times \text { Sample } \\
\text { Original })\end{array}$ & $\begin{array}{c}\text { Data Dimension } \\
\text { Gene } \times \text { Sample } \\
(\text { Used })\end{array}$ & $\begin{array}{c}\text { Sample } \\
\text { Class } \\
\text { Labels }\end{array}$ \\
\hline Leukemia & $7129 \times 72$ & $7070 \times 72$ & 2 & Breast & $7129 \times 49$ & $7129 \times 49$ & 2 \\
\hline Colon & $2000 \times 62$ & $2000 \times 62$ & 2 & MLL & $12582 \times 72$ & $12582 \times 72$ & 3 \\
\hline Prostate & $12600 \times 136$ & $12600 \times 136$ & 2 & SRBCT & $2308 \times 63$ & $2308 \times 63$ & 4 \\
\hline Lung & $12533 \times 181$ & $12533 \times 181$ & 2 & RAHC & $41057 \times 50$ & $41057 \times 50$ & 2 \\
\hline Rbreast & $24481 \times 97$ & $24188 \times 97$ & 2 & RAOA & $18433 \times 30$ & $18433 \times 30$ & 2 \\
\hline
\end{tabular}

2

4

5

6

7 


\section{Table 2 (on next page)}

Classification Accuracy of MFSAC-EC depending on varying number of genes selected by each Filter

This table shows the impact of parameter $\mathrm{P}$ with respect to sample classification accuracy (\%) in terms of both LOOCV and 10-Fold Cross Validation approach. P defines the number of top ranked genes selected by each filter method. 
Table 2: Classification Accuracy of MFSAC-EC depending on varying number of genes selected by each Filter

\begin{tabular}{|c|c|c|c|c|c|c|c|c|c|c|c|c|c|c|c|c|c|c|c|c|c|c|c|c|c|}
\hline \multirow{3}{*}{ Dataset } & \multirow{3}{*}{$\begin{array}{c}\text { Evaluation } \\
\text { Metric }\end{array}$} & \multicolumn{24}{|c|}{ MFSAC-EC } \\
\hline & & \multicolumn{4}{|c|}{$\mathrm{P}=100$} & \multicolumn{4}{|c|}{$\mathrm{P}=200$} & \multicolumn{4}{|c|}{$\mathrm{P}=500$} & \multicolumn{4}{|c|}{$\mathrm{P}=1000$} & \multicolumn{4}{|c|}{$\mathrm{P}=1200$} & \multicolumn{4}{|c|}{$\mathrm{P}=1500$} \\
\hline & & NB & KNN & DT & SVM & NB & KNN & DT & SVM & NB & KNN & DT & SVM & NB & KNN & DT & SVM & NB & KNN & DT & SVM & NB & KNN & DT & SVM \\
\hline \multirow{2}{*}{ Leukemia } & LOOCV & 98.6 & 98.6 & 98.6 & 98.6 & 98.6 & 98.6 & 98.6 & 98.6 & 100 & 100 & 100 & 100 & 100 & 100 & 100 & 100 & 100 & 100 & 100 & 100 & 100 & 100 & 100 & 100 \\
\hline & 10 Fold & 98.6 & 98.6 & 98.6 & 98.6 & 98.6 & 98.6 & 98.6 & 98.6 & 100 & 100 & 100 & 100 & 100 & 100 & 100 & 100 & 100 & 100 & 100 & 100 & 100 & 100 & 100 & 100 \\
\hline \multirow{2}{*}{ RAHC } & LOOCV & 100 & 100 & 100 & 100 & 100 & 100 & 100 & 100 & 100 & 100 & 100 & 100 & 100 & 100 & 100 & 100 & 100 & 100 & 100 & 100 & 100 & 100 & 100 & 100 \\
\hline & 10 Fold & 100 & 100 & 100 & 100 & 100 & 100 & 100 & 100 & 100 & 100 & 100 & 100 & 100 & 100 & 100 & 100 & 100 & 100 & 100 & 100 & 100 & 100 & 100 & 100 \\
\hline \multirow{2}{*}{ MLL } & LOOCV & 98.6 & 100 & 100 & 97.2 & 100 & 100 & 100 & 100 & 100 & 100 & 100 & 100 & 100 & 100 & 100 & 100 & 100 & 100 & 100 & 100 & 100 & 100 & 100 & 100 \\
\hline & 10 Fold & 97.2 & 100 & 100 & 97.2 & 100 & 100 & 100 & 100 & 100 & 100 & 100 & 100 & 100 & 100 & 100 & 100 & 100 & 100 & 100 & 100 & 100 & 100 & 100 & 100 \\
\hline \multirow{2}{*}{ RAOA } & LOOCV & 100 & 100 & 100 & 100 & 100 & 100 & 100 & 100 & 100 & 100 & 100 & 100 & 100 & 100 & 100 & 100 & 100 & 100 & 100 & 100 & 100 & 100 & 100 & 100 \\
\hline & 10 Fold & 100 & 100 & 100 & 100 & 100 & 100 & 100 & 100 & 100 & 100 & 100 & 100 & 100 & 100 & 100 & 100 & 100 & 100 & 100 & 100 & 100 & 100 & 100 & 100 \\
\hline \multirow{2}{*}{ SRBCT } & LOOCV & 98.4 & 98.4 & 100 & 98.4 & 100 & 100 & 100 & 100 & 100 & 100 & 100 & 100 & 100 & 100 & 100 & 100 & 100 & 100 & 100 & 100 & 100 & 100 & 100 & 100 \\
\hline & 10 Fold & 100 & 98.4 & 98.4 & 98.4 & 100 & 100 & 100 & 100 & 100 & 100 & 100 & 100 & 100 & 100 & 100 & 100 & 100 & 100 & 100 & 100 & 100 & 100 & 100 & 100 \\
\hline \multirow{2}{*}{ Breast } & LOOCV & 98 & 95.9 & 93.9 & 95.9 & 100 & 100 & 100 & 100 & 100 & 100 & 100 & 100 & 100 & 100 & 100 & 100 & 100 & 100 & 100 & 100 & 100 & 100 & 100 & 100 \\
\hline & 10 Fold & 100 & 95.9 & 95.9 & 98 & 100 & 100 & 100 & 100 & 100 & 100 & 100 & 100 & 100 & 100 & 100 & 100 & 100 & 100 & 100 & 100 & 100 & 100 & 100 & 100 \\
\hline \multirow{2}{*}{ Lung } & LOOCV & 100 & 100 & 100 & 100 & 100 & 100 & 100 & 100 & 100 & 100 & 100 & 100 & 100 & 100 & 100 & 100 & 100 & 100 & 100 & 100 & 100 & 100 & 100 & 100 \\
\hline & 10 Fold & 100 & 100 & 100 & 100 & 100 & 100 & 100 & 100 & 100 & 100 & 100 & 100 & 100 & 100 & 100 & 100 & 100 & 100 & 100 & 100 & 100 & 100 & 100 & 100 \\
\hline \multirow{2}{*}{ Rbreast } & LOOCV & 92.6 & 93.7 & 93.7 & 95.8 & 99 & 97.9 & 100 & 99 & 100 & 100 & 100 & 100 & 100 & 100 & 100 & 100 & 100 & 100 & 100 & 100 & 100 & 100 & 100 & 100 \\
\hline & 10 Fold & 91.6 & 96.8 & 95.8 & 97.9 & 97.9 & 99 & 99 & 99 & 100 & 100 & 100 & 100 & 100 & 100 & 100 & 100 & 100 & 100 & 100 & 100 & 100 & 100 & 100 & 100 \\
\hline \multirow{2}{*}{ COLON } & LOOCV & 91.9 & 91.9 & 93.6 & 91.9 & 91.9 & 91.9 & 96.8 & 91.9 & 98.4 & 98.4 & 96.8 & 98.4 & 98.4 & 98.4 & 98.4 & 98.4 & 98.4 & 98.4 & 98.4 & 100 & 100 & 100 & 98.4 & 100 \\
\hline & 10 Fold & 91.9 & 91.9 & 93.6 & 91.9 & 91.9 & 91.9 & 95.2 & 91.9 & 98.4 & 96.8 & 96.8 & 96.8 & \begin{tabular}{|l|}
98.4 \\
\end{tabular} & 100 & 98.4 & 98.4 & 100 & 98.4 & 98.4 & 100 & 100 & 100 & 98.4 & 100 \\
\hline \multirow{2}{*}{ Prostrate } & LOOCV & 83.8 & 90.4 & 92.7 & 86.8 & 88.2 & 92.7 & 92.7 & 88.2 & 91.2 & 95.6 & 97.1 & 91.9 & 94.9 & 97.1 & 97.8 & 94.1 & 98.5 & 98.5 & 97.8 & 98.5 & 98.5 & 99.3 & 98.5 & 99.3 \\
\hline & 10 Fold & 85.3 & 88.2 & 91.9 & 86.8 & 88.2 & 91.9 & \begin{tabular}{|l|}
93.4 \\
\end{tabular} & 89.7 & 91.9 & 95.6 & 97.8 & 91.2 & 95.6 & 97.8 & 97.8 & 94.1 & 99.3 & 98.5 & 97.8 & 98.5 & 99.3 & 99.3 & 97.8 & 99.3 \\
\hline
\end{tabular}

2 


\section{Table 3(on next page)}

Total Execution Time in a single run of MFSAC-EC on Different Datasets

Total execution time in a single run of MFSAC-EC including Bootstrapped dataset creation, Feature Selection by filter methods and supervised attribute clustering approach, Training, Testing using LOOCV, 5-Fold, 10-Fold, and Random Splitting is given in the first row. While execution time using only 10-Fold Cross Validation is given in the 2 nd row. Here the time for the best $P$ value is shown here. 
Table 3: Total Execution Time (Bootstrapping, Feature Selection, Training, Testing for LOOCV, 5-Fold, 10-Fold, Random Splitting) in a single run of MFSAC-EC on Different Datasets

\begin{tabular}{|c|c|c|c|c|c|c|c|c|c|c|}
\hline & Leukemia & RAHC & MLL & RAOA & SRBCT & Breast & Lung & Rbreast & COLON & Prostrate \\
\hline No. of Feature selected for best result & 500 & 100 & 200 & 100 & 200 & 200 & 100 & 500 & 1200 & 3000 \\
\hline Total Time Taken & $8 \mathrm{mins} 23 \mathrm{secs}$ & $\begin{array}{c}7 \mathrm{mins} \\
32 \mathrm{secs}\end{array}$ & $\begin{array}{l}7 \mathrm{mins} \\
54 \mathrm{secs}\end{array}$ & $\begin{array}{l}4 \mathrm{mins} \\
43 \mathrm{secs}\end{array}$ & $\begin{array}{l}5 \mathrm{mins} \\
17 \mathrm{secs}\end{array}$ & $\begin{array}{l}4 \operatorname{mins} \\
2 \text { secs }\end{array}$ & $\begin{array}{l}11 \mathrm{mins} \\
14 \mathrm{secs}\end{array}$ & $\begin{array}{l}10 \mathrm{mins} \\
22 \mathrm{secs}\end{array}$ & $\begin{array}{l}17 \mathrm{mins} \\
40 \mathrm{secs}\end{array}$ & $\begin{array}{c}1 \mathrm{hr} 18 \mathrm{mins} \\
41 \mathrm{secs}\end{array}$ \\
\hline Time Taken for only 10 fold & $35 \mathrm{secs}$ & 30 secs & 41 secs & $36 \mathrm{secs}$ & 30 secs & $32 \operatorname{secs}$ & 36 secs & $33 \operatorname{secs}$ & 30 secs & 36 secs \\
\hline
\end{tabular}




\section{Table 4 (on next page)}

Classification Accuracy of the proposed MFSAC-EC model with respect to LOOCV

Classification accuracy (\%) of MFSAC-EC model has been shown in terms of LOOCV with respect to four ensemble classifiers MFSAC-EC + NB, MFSAC-EC+KNN, MFSAC-EC+DT, and MFSAC-EC+SVM. Every ensemble classifier is run 50 times using LOOCV for every dataset and the accuracy is shown which is obtained maximum number of times. 
1

2

Table 4. Classification Accuracy of the proposed MFSAC-EC model with respect to LOOCV

\begin{tabular}{|c|c|c|c|c|c|c|c|c|c|c|c|}
\hline \multirow{2}{*}{ Dataset } & \multirow{2}{*}{\multicolumn{2}{|c|}{ Proposed Model }} & \multicolumn{3}{|c|}{ Cluster Representatives } & \multirow{2}{*}{ Dataset } & \multicolumn{2}{|c|}{ Proposed Model } & \multicolumn{3}{|c|}{ Cluster Representatives } \\
\hline & & & 1 & 2 & 3 & & & & 1 & 2 & 3 \\
\hline \multirow{4}{*}{ COLON } & \multirow{20}{*}{$\begin{array}{c}\text { MFSA } \\
\text { C-EC }\end{array}$} & NB & 100 & 98.39 & 98.39 & \multirow{4}{*}{ MLL } & \multirow{20}{*}{$\begin{array}{c}\text { MFSAC- } \\
\text { EC }\end{array}$} & NB & 100 & 100 & 100 \\
\hline & & $\mathrm{KNN}$ & 98.39 & 100 & 100 & & & $\mathrm{KNN}$ & 100 & 100 & 100 \\
\hline & & DT & 98.39 & 98.39 & 98.39 & & & DT & 100 & 100 & 100 \\
\hline & & SVM & 100 & 98.4 & 98.4 & & & SVM & 100 & 100 & 100 \\
\hline \multirow{4}{*}{ Prostate } & & NB & 97.06 & 97.79 & 98.53 & \multirow{4}{*}{ SRBCT } & & NB & 96.83 & 100 & 100 \\
\hline & & KNN & 97.79 & 97.79 & 98.53 & & & KNN & 96.83 & 100 & 100 \\
\hline & & DT & 97.79 & 98.53 & 97.79 & & & DT & 96.83 & 98.41 & 100 \\
\hline & & SVM & 98.53 & 99.26 & 99.26 & & & SVM & 82.54 & 98.41 & 100 \\
\hline \multirow{4}{*}{ Leukemia } & & NB & 100 & 100 & 100 & \multirow{4}{*}{ Lung } & & $\mathrm{NB}$ & 100 & 100 & 100 \\
\hline & & KNN & 100 & 100 & 100 & & & KNN & 100 & 100 & 100 \\
\hline & & DT & 100 & 100 & 100 & & & DT & 100 & 100 & 100 \\
\hline & & SVM & 100 & 100 & 100 & & & SVM & 100 & 100 & 100 \\
\hline \multirow{8}{*}{ Breast } & & NB & 100 & 100 & 100 & \multirow{4}{*}{ RAHC } & & NB & 100 & 100 & 100 \\
\hline & & KNN & 100 & 100 & 100 & & & KNN & 100 & 100 & 100 \\
\hline & & DT & 100 & 100 & 100 & & & DT & 100 & 100 & 100 \\
\hline & & SVM & 100 & 100 & 100 & & & SVM & 100 & 100 & 100 \\
\hline & & NB & 100 & 100 & 100 & \multirow{4}{*}{ RBreast } & & NB & 100 & 100 & 100 \\
\hline & & KNN & 100 & 100 & 100 & & & KNN & 100 & 100 & 100 \\
\hline & & DT & 100 & 100 & 100 & & & DT & 100 & 100 & 100 \\
\hline & & SVM & 100 & 100 & 100 & & & SVM & 100 & 100 & 100 \\
\hline
\end{tabular}

3 


\section{Table 5 (on next page)}

Classification Accuracy of the proposed MFSAC-EC model with respect to 5-Fold Cross Validation

Classification accuracy (\%) of MFSAC-EC model has been shown in terms of 5-Fold Cross Validation with respect to four ensemble classifiers MFSAC-EC + NB, MFSAC-EC+KNN, MFSAC-EC+DT, and MFSAC-EC+SVM. Every ensemble classifier is run 50 times using 5-Fold Cross Validation for every dataset and the accuracy is shown which is obtained maximum number of times. 
1

Table 5. Classification Accuracy of the proposed MFSAC-EC model with respect to 5-Fold Cross Validation

\begin{tabular}{|c|c|c|c|c|c|c|c|c|c|c|c|}
\hline \multirow[t]{2}{*}{ Dataset } & \multirow{2}{*}{\multicolumn{2}{|c|}{ Proposed Model }} & \multicolumn{3}{|c|}{ Cluster Representatives } & \multirow[t]{2}{*}{ Dataset } & \multicolumn{2}{|c|}{ Proposed Model } & \multicolumn{3}{|c|}{ Cluster Representatives } \\
\hline & & & 1 & 2 & 3 & & & & 1 & 2 & 3 \\
\hline \multirow{4}{*}{ COLON } & \multirow{20}{*}{ MFSAC-EC } & NB & 96.77 & 96.77 & 96.77 & \multirow{4}{*}{ MLL } & \multirow{20}{*}{$\begin{array}{c}\text { MFSAC } \\
\text {-EC }\end{array}$} & NB & 100 & 100 & 100 \\
\hline & & KNN & 98.39 & 96.77 & 96.77 & & & KNN & 98.61 & 100 & 100 \\
\hline & & DT & 98.39 & 96.77 & 98.39 & & & DT & 98.61 & 100 & 100 \\
\hline & & SVM & 98.39 & 96.77 & 96.77 & & & SVM & 100 & 100 & 100 \\
\hline \multirow{4}{*}{ Prostate } & & NB & 97.06 & 97.79 & 98.53 & \multirow{4}{*}{ SRBCT } & & NB & 98.41 & 100 & 100 \\
\hline & & KNN & 97.79 & 97.79 & 99.26 & & & KNN & 96.83 & 100 & 100 \\
\hline & & DT & 97.06 & 97.79 & 94.85 & & & DT & 96.83 & 98.41 & 100 \\
\hline & & SVM & 97.79 & 98.53 & 99.26 & & & SVM & 96.83 & 100 & 100 \\
\hline \multirow{4}{*}{ Leukemia } & & NB & 100 & 100 & 100 & \multirow{4}{*}{ Lung } & & $\mathrm{NB}$ & 100 & 100 & 100 \\
\hline & & KNN & 100 & 100 & 100 & & & KNN & 100 & 100 & 100 \\
\hline & & DT & 100 & 100 & 100 & & & DT & 100 & 99.44 & 100 \\
\hline & & SVM & 100 & 100 & 100 & & & SVM & 100 & 100 & 100 \\
\hline \multirow{8}{*}{ Breast } & & NB & 100 & 100 & 100 & \multirow{4}{*}{ RAHC } & & $\mathrm{NB}$ & 100 & 100 & 100 \\
\hline & & KNN & 100 & 100 & 100 & & & KNN & 100 & 100 & 100 \\
\hline & & DT & 100 & 100 & 100 & & & DT & 100 & 100 & 100 \\
\hline & & SVM & 100 & 100 & 100 & & & SVM & 100 & 100 & 100 \\
\hline & & NB & 100 & 100 & 100 & \multirow{4}{*}{ RBreast } & & NB & 100 & 100 & 100 \\
\hline & & KNN & 100 & 100 & 100 & & & KNN & 100 & 100 & 100 \\
\hline & & DT & 100 & 100 & 100 & & & DT & 100 & 100 & 100 \\
\hline & & SVM & 100 & 100 & 100 & & & SVM & 100 & 100 & 100 \\
\hline
\end{tabular}

3 


\section{Table 6(on next page)}

Classification Accuracy of the proposed MFSAC-EC model with respect to 10-Fold Cross Validation

Classification accuracy (\%) of MFSAC-EC model has been shown in terms of 10-Fold Cross Validation with respect to four ensemble classifiers MFSAC-EC + NB, MFSAC-EC+KNN, MFSAC-EC+DT, and MFSAC-EC+SVM. Every ensemble classifier is run 50 times using 10-Fold Cross Validation for every dataset and the accuracy is shown which is obtained maximum number of times. 
1

Table 6. Classification Accuracy of the proposed MFSAC-EC model with respect to 10-Fold Cross Validation

\begin{tabular}{|c|c|c|c|c|c|c|c|c|c|c|c|}
\hline \multirow{2}{*}{ Dataset } & \multirow{2}{*}{\multicolumn{2}{|c|}{ Proposed Model }} & \multicolumn{3}{|c|}{ Cluster Representatives } & \multirow{2}{*}{ Dataset } & \multirow{2}{*}{\multicolumn{2}{|c|}{ Proposed Model }} & \multicolumn{3}{|c|}{ Cluster Representatives } \\
\hline & & & \multirow{2}{*}{$\begin{array}{c}1 \\
98.39\end{array}$} & \multirow{2}{*}{$\begin{array}{c}2 \\
98.39 \\
\end{array}$} & \multirow{2}{*}{$\begin{array}{c}3 \\
98.39\end{array}$} & & & & 1 & 2 & 3 \\
\hline \multirow{4}{*}{ COLON } & \multirow{20}{*}{ MFSAC-EC } & NB & & & & \multirow{4}{*}{ MLL } & \multirow{20}{*}{ MFSAC-EC } & NB & 100 & 100 & 100 \\
\hline & & KNN & 98.39 & 98.39 & 100 & & & KNN & 100 & 100 & 100 \\
\hline & & DT & 98.39 & 98.39 & 98.39 & & & DT & 100 & 100 & 100 \\
\hline & & SVM & 98.39 & 98.39 & 98.39 & & & SVM & 100 & 100 & 100 \\
\hline \multirow{4}{*}{ Prostate } & & NB & 97.06 & 97.79 & 98.53 & \multirow{4}{*}{ SRBCT } & & NB & 96.83 & 96.83 & 100 \\
\hline & & KNN & 97.79 & 97.79 & 99.26 & & & KNN & 92.06 & 100 & 100 \\
\hline & & DT & 97.06 & 97.79 & 94.85 & & & DT & 95.24 & 96.83 & 100 \\
\hline & & SVM & 97.79 & 98.53 & 99.26 & & & SVM & 80.95 & 92.06 & 100 \\
\hline \multirow{4}{*}{ Leukemia } & & $\mathrm{NB}$ & 100 & 100 & 100 & \multirow{4}{*}{ Lung } & & NB & 100 & 100 & 100 \\
\hline & & KNN & 100 & 100 & 100 & & & KNN & 100 & 100 & 100 \\
\hline & & DT & 100 & 100 & 100 & & & DT & 100 & 100 & 100 \\
\hline & & SVM & 100 & 100 & 100 & & & SVM & 100 & 100 & 100 \\
\hline \multirow{4}{*}{ Breast } & & NB & 100 & 100 & 100 & \multirow{4}{*}{ RBreast } & & NB & 100 & 100 & 100 \\
\hline & & KNN & 100 & 100 & 100 & & & KNN & 100 & 100 & 100 \\
\hline & & DT & 100 & 100 & 100 & & & DT & 100 & 100 & 100 \\
\hline & & SVM & 100 & 100 & 100 & & & SVM & 100 & 100 & 100 \\
\hline \multirow{4}{*}{ RAOA } & & NB & 100 & 100 & 100 & \multirow{4}{*}{ RAHC } & & NB & 100 & 100 & 100 \\
\hline & & KNN & 100 & 100 & 100 & & & KNN & 100 & 100 & 100 \\
\hline & & DT & 100 & 100 & 100 & & & DT & 100 & 100 & 100 \\
\hline & & SVM & 100 & 100 & 100 & & & SVM & 100 & 100 & 100 \\
\hline
\end{tabular}

2 


\section{Table 7 (on next page)}

Classification Accuracy of the proposed MFSAC-EC model with respect to Random Splitting of the Datasets

Classification accuracy (\%) of MFSAC-EC model has been shown in terms of random splitting with respect to four ensemble classifiers MFSAC-EC + NB, MFSAC-EC+KNN, MFSAC-EC+DT, and MFSAC-EC+SVM. Every ensemble classifier is run 50 times using random splitting for every dataset and the accuracy is shown which is obtained maximum number of times. For random splitting the dataset is divided into training (2/3) and testing (1/3) part 50 times randomly. 
1

2

Table 7. Classification Accuracy of the proposed MFSAC-EC model with respect to Random Splitting of the Datasets

\begin{tabular}{|c|c|c|c|c|c|c|c|c|c|c|c|}
\hline \multirow{2}{*}{ Dataset } & \multirow{2}{*}{\multicolumn{2}{|c|}{ Proposed Model }} & \multicolumn{3}{|c|}{ Cluster Representatives } & \multirow{2}{*}{ Dataset } & \multirow{2}{*}{\multicolumn{2}{|c|}{ Proposed Model }} & \multicolumn{3}{|c|}{ Cluster Representatives } \\
\hline & & & \multirow{2}{*}{$\begin{array}{c}1 \\
98.39\end{array}$} & \multirow{2}{*}{$\begin{array}{c}2 \\
98.39\end{array}$} & \multirow{2}{*}{$\begin{array}{c}3 \\
98.39\end{array}$} & & & & 1 & 2 & 3 \\
\hline \multirow{4}{*}{ COLON } & \multirow{20}{*}{$\begin{array}{c}\text { MFSAC- } \\
\text { EC }\end{array}$} & NB & & & & \multirow{4}{*}{ MLL } & \multirow{20}{*}{$\begin{array}{c}\text { MFSAC- } \\
\text { EC }\end{array}$} & NB & 100 & 100 & 100 \\
\hline & & KNN & 98.39 & 98.39 & 98.39 & & & KNN & 100 & 100 & 100 \\
\hline & & DT & 98.39 & 98.39 & 98.39 & & & DT & 98.61 & 100 & 98.61 \\
\hline & & SVM & 98.39 & 100 & 98.39 & & & SVM & 100 & 100 & 100 \\
\hline \multirow{4}{*}{ Prostate } & & NB & 94.68 & 95.74 & 93.62 & \multirow{4}{*}{ SRBCT } & & NB & 95 & 85 & 95 \\
\hline & & KNN & 97.87 & 96.81 & 92.55 & & & KNN & 95 & 100 & 90 \\
\hline & & DT & 94.68 & 94.68 & 94.68 & & & DT & 80 & 90 & 95 \\
\hline & & SVM & 94.68 & 96.81 & 94.68 & & & SVM & 65 & 75 & 95 \\
\hline \multirow{4}{*}{ Leukemia } & & NB & 100 & 100 & 100 & \multirow{4}{*}{ Lung } & & NB & 100 & 100 & 100 \\
\hline & & KNN & 100 & 100 & 100 & & & KNN & 100 & 100 & 100 \\
\hline & & DT & 100 & 100 & 100 & & & DT & 100 & 100 & 100 \\
\hline & & SVM & 100 & 100 & 100 & & & SVM & 100 & 100 & 100 \\
\hline \multirow{4}{*}{ RAOA } & & NB & 100 & 100 & 100 & \multirow{4}{*}{ RAHC } & & NB & 100 & 100 & 100 \\
\hline & & KNN & 100 & 100 & 100 & & & KNN & 100 & 100 & 100 \\
\hline & & DT & 100 & 100 & 100 & & & DT & 100 & 100 & 81.25 \\
\hline & & SVM & 100 & 100 & 100 & & & SVM & 100 & 100 & 81.25 \\
\hline \multirow{4}{*}{ Breast } & & NB & 100 & 100 & 100 & \multirow{4}{*}{ RBreast } & & NB & 91.94 & 91.94 & 91.94 \\
\hline & & KNN & 100 & 100 & 100 & & & KNN & 85.48 & 87.10 & 83.87 \\
\hline & & DT & 100 & 100 & 100 & & & DT & 83.87 & 79.03 & 80.65 \\
\hline & & SVM & 100 & 100 & 100 & & & SVM & 93.55 & 91.94 & 91.94 \\
\hline
\end{tabular}

3 


\section{Table 8(on next page)}

Evaluation of MFSAC-EC classifier based on SN, SP, PPV, NPV, FPR for two class data sets with respect to LOOCV

The performance of the MFSAC-EC model for two class datasets is represented using Receiver Operator Characteristic (ROC) analysis. SN represents Sensitivity, SP represents Specificity, PPV represents Positive Predicted Value, NPV represents Negative Predicted Value, and FPR represents False Positive Rate. 
$\frac{1}{2}$

Table 8. Evaluation of MFSAC-EC classifier based on SN, SP, PPV, NPV, FPR for two class data sets with respect to LOOCV

\begin{tabular}{|c|c|c|c|c|c|c|c|c|c|c|c|c|c|c|c|}
\hline Dataset & \multicolumn{2}{|c|}{ Proposed Model } & SN & SP & PPV & NPV & FPR & Dataset & \multicolumn{2}{|c|}{ Proposed Model } & SN & SP & PPV & NPV & FPR \\
\hline \multirow{4}{*}{ Leukemia } & \multirow{16}{*}{$\begin{array}{c}\text { MFSGC- } \\
\text { EC }\end{array}$} & NB & 100 & 100 & 100 & 100 & 0 & \multirow{4}{*}{ Breast } & \multirow{16}{*}{$\begin{array}{c}\text { MFSGC- } \\
\text { EC }\end{array}$} & NB & 100 & 100 & 100 & 100 & 0 \\
\hline & & KNN & 100 & 100 & 100 & 100 & 0 & & & KNN & 100 & 100 & 100 & 100 & 0 \\
\hline & & DT & 100 & 100 & 100 & 100 & 0 & & & DT & 100 & 100 & 100 & 100 & 0 \\
\hline & & SVM & 100 & 100 & 100 & 100 & 0 & & & SVM & 100 & 100 & 100 & 100 & 0 \\
\hline \multirow{4}{*}{ Prostate } & & NB & 98.7 & 98.3 & 98.7 & 98.3 & 1.7 & \multirow{4}{*}{ Rbreast } & & NB & 100 & 100 & 100 & 100 & 0 \\
\hline & & KNN & 98.7 & 98.3 & 98.7 & 98.3 & 1.7 & & & KNN & 100 & 100 & 100 & 100 & 0 \\
\hline & & DT & 100 & 96.61 & 97.46 & 100 & 3.4 & & & DT & 100 & 100 & 100 & 100 & 0 \\
\hline & & SVM & 100 & 98.3 & 98.7 & 100 & 1.7 & & & SVM & 100 & 100 & 100 & 100 & 0 \\
\hline \multirow{4}{*}{ Colon } & & NB & 100 & 100 & 100 & 100 & 0 & \multirow{4}{*}{ Lung } & & NB & 100 & 100 & 100 & 100 & 0 \\
\hline & & KNN & 100 & 100 & 100 & 100 & 0 & & & KNN & 100 & 100 & 100 & 100 & 0 \\
\hline & & DT & 100 & 100 & 100 & 100 & 0 & & & DT & 100 & 100 & 100 & 100 & 0 \\
\hline & & SVM & 100 & 100 & 100 & 100 & 0 & & & SVM & 100 & 100 & 100 & 100 & 0 \\
\hline \multirow{4}{*}{ RAHC } & & NB & 100 & 100 & 100 & 100 & 0 & \multirow{4}{*}{ RAOA } & & NB & 100 & 100 & 100 & 100 & 0 \\
\hline & & KNN & 100 & 100 & 100 & 100 & 0 & & & KNN & 100 & 100 & 100 & 100 & 0 \\
\hline & & DT & 100 & 100 & 100 & 100 & 0 & & & DT & 100 & 100 & 100 & 100 & 0 \\
\hline & & SVM & 100 & 100 & 100 & 100 & 0 & & & SVM & 100 & 100 & 100 & 100 & 0 \\
\hline
\end{tabular}

3 


\section{Table 9 (on next page)}

Comparison of MFSAC-EC + DT with different existing Ensemble Classifiers using DT in terms of 10-Fold Cross Validation

Here MFSAC-EC + DT model is compared with existing ensemble classifiers where DT is used as base classifier. C4.5 algorithm is used as DT. 
1

2 Table 9. Comparison of MFSAC-EC using DT with different existing Ensemble Classifiers using DT in terms of 10-Fold Cross Validation

\begin{tabular}{|c|c|c|c|c|c|c|c|c|c|}
\hline & $\begin{array}{c}\text { MFSAC- } \\
\text { EC }\end{array}$ & $\begin{array}{c}\text { PCA- } \\
\text { based } \\
\text { RotBoost }\end{array}$ & $\begin{array}{c}\text { ICA-based } \\
\text { RotBoost }\end{array}$ & AdaBoost & Bagging & Arcing & $\begin{array}{c}\text { Rotation } \\
\text { Forest }\end{array}$ & EN-NEW1 & EN-NEW2 \\
\hline Colon & $\mathbf{9 8 . 3 9}$ & 95.48 & 96.1 & 94.97 & 94.92 & 69.35 & 95.21 & 79.03 & 83.87 \\
\hline Leukemia & $\mathbf{1 0 0}$ & 98.75 & 98.77 & 98.22 & 97.47 & Not Found & 97.97 & Not Found & Not Found \\
\hline Breast & $\mathbf{1 0 0}$ & 94.39 & 97.88 & 98.89 & 92.74 & 80.41 & 98.6 & 94.85 & 95.88 \\
\hline Lung & $\mathbf{1 0 0}$ & 98.11 & 99.54 & 96.3 & 97.08 & 97.24 & 97.56 & 98.34 & 99.45 \\
\hline Prostate & $\mathbf{9 7 . 7 9}$ & Not Found & Not Found & 90.44 & 94.12 & 87.5 & Not Found & 94.85 & 97.06 \\
\hline MLL & $\mathbf{1 0 0}$ & 98.86 & 99.31 & 97.63 & 97.11 & 91.67 & 97.61 & 93.06 & 98.61 \\
\hline SRBCT & $\mathbf{1 0 0}$ & 99.5 & 99.59 & 98.16 & 96.46 & Not Found & 97.44 & Not Found & Not Found \\
\hline
\end{tabular}

3 


\section{Table $\mathbf{1 0}$ (on next page)}

Comparison of MFSAC-EC using DT, KNN, NB, SVM with different existing Ensemble Classifiers using DT, KNN, NB, SVM in terms of 10-Fold Cross Validation

Here classification accuracy (\%) of four ensemble classifiers MFSAC-EC + NB, MFSAC-EC +

KNN, MFSAC-EC+DT, and MFSAC-EC+SVM are shown with respect to results of other existing ensemble classifiers with the same base learners. The best accuracy (\%) for every dataset is shown in bold. 
Table 10. Comparison of MFSAC-EC using DT, KNN, NB, SVM with different existing Ensemble Classifiers using DT, KNN, NB, SVM 2 in terms of 10-Fold Cross Validation

\begin{tabular}{|c|c|c|c|c|c|c|c|c|c|c|c|c|c|c|c|c|c|}
\hline \multirow{2}{*}{ Dataset } & \multicolumn{4}{|c|}{ MFSAC-EC } & \multicolumn{3}{|c|}{ Bagging } & \multicolumn{3}{|c|}{ Boosting } & \multicolumn{3}{|c|}{ Stacking } & \multicolumn{2}{|c|}{ HBSA } & \multirow{2}{*}{ SD_Ens } & \multirow{2}{*}{ Meta_Ens } \\
\hline & DT & NB & KNN & SVM & DT & NB & KNN & DT & NB & KNN & DT & NB & KNN & KNN & SVM & & \\
\hline Leukemia & 100 & 100 & 100 & 100 & 94.12 & 88.23 & 73.53 & 91.18 & 88.24 & 75.53 & 91.18 & 91.18 & 91.18 & 88.46 & 88.46 & 92.45 & 94.12 \\
\hline Colon & 98.39 & 98.39 & 100 & 98.39 & 95.16 & 66.13 & 90.32 & 98.39 & 87.1 & 91.94 & 98.39 & 93.59 & 93.59 & 75 & 85 & 94.4 & 99.21 \\
\hline Prostate & 97.79 & 99.26 & 99.26 & 99.26 & 26.47 & 26.47 & 38.24 & 26.47 & 26.47 & 52.94 & 26.47 & 26.47 & 52.94 & 85.29 & 97.06 & 52.94 & 52.94 \\
\hline Lung & 100 & 100 & 100 & 100 & 91.28 & 96.64 & 97.32 & 81.88 & 95.3 & 97.99 & 97.99 & 97.99 & 96.64 & $\begin{array}{c}\text { Not } \\
\text { Found }\end{array}$ & Not Found & 81.88 & 97.99 \\
\hline Breast & 100 & 100 & 100 & 100 & 78.95 & 36.84 & 68.42 & 68.42 & 36.84 & 68.42 & 68.42 & 68.42 & 68.42 & $\begin{array}{c}\text { Not } \\
\text { Found }\end{array}$ & Not Found & 73.49 & 79.87 \\
\hline
\end{tabular}




\section{Table 11 (on next page)}

Comparison of MFSAC-EC using SVM and KNN with respect to different existing deep learning Classifiers using random splitting

Here classification accuracy (\%) of two ensemble classifiers MFSAC-EC + KNN, and MFSAC$\mathrm{EC}+\mathrm{SVM}$ are shown with respect to results of other existing ensemble classifiers with the same base learners. The best accuracy (\%) for every dataset is shown in bold. 
Table 11. Comparison of MFSAC-EC using SVM and KNN with respect to different existing deep learning Classifiers using random splitting

\begin{tabular}{|c|c|c|c|c|c|c|}
\hline \multirow{2}{*}{ Dataset } & \multicolumn{3}{|c|}{ SVM } & \multicolumn{3}{c|}{ KNN } \\
\cline { 2 - 7 } & MFSAC-EC & Folded Autoencoder & Autoencoder & MFSAC-EC & Folded Autoencoder & Autoencoder \\
\hline Colon & $\mathbf{1 0 0}$ & 90.15 & 73.11 & $\mathbf{9 8 . 3 9}$ & 81.09 & 56.97 \\
\hline Prostate & $\mathbf{9 6 . 8 1}$ & 84.16 & 64.3 & $\mathbf{9 7 . 8 7}$ & 76.48 & 52.1 \\
\hline Leukemia & $\mathbf{1 0 0}$ & 93.62 & 84.12 & $\mathbf{1 0 0}$ & 85.24 & 77.13 \\
\hline
\end{tabular}




\section{Table 12 (on next page)}

List of genes selected by MFSAC-EC model for Colon and Leukemia cancer Datasets

Here second column represents the gene names while third column indicate the gene accession number. The fourth column indicates the description of the gene while the fifth column indicates the literature where it has been referred as cancer biomarker. 
1

2

Table 12. List of genes selected by MFSAC-EC model for Colon and Leukemia cancer Datasets

\begin{tabular}{|c|c|c|c|c|}
\hline Dataset & Gene Name & Accession Number & Description & Validation of Genes \\
\hline \multirow{8}{*}{ Colon } & TPM1 & Hsa.1130 & Human tropomyosin isoform mRNA, complete cds. & $\begin{array}{l}\text { Gardina 2006, Thorsen } \\
\text { 2008, Botchkina } 2009\end{array}$ \\
\hline & IGFBP4 & Hsa.1532 & $\begin{array}{l}\text { Human insulin-like growth factor binding protein-4 } \\
\text { (IGFBP4) gene, promoter and complete cds. }\end{array}$ & $\begin{array}{l}\text { Durai 2007, Singh 1994, } \\
\text { Yu } 2000\end{array}$ \\
\hline & MYL9 & Hsa.1832 & $\begin{array}{l}\text { Myosin Regulatory Light Chain 2, Smooth Muscle } \\
\text { Isoform (Human); contains element TAR1 repetitive } \\
\text { element }\end{array}$ & Yan 2012, Zhu 2019 \\
\hline & ALDH1L1 & Hsa.10224 & $\begin{array}{l}\text { Aldehyde Dehydrogenase, Mitochodrial X Precursor } \\
\text { (Homo sapiens) }\end{array}$ & $\begin{array}{c}\text { Feng 2018, Waals 2018, } \\
\text { Kozovska } 2018 \\
\end{array}$ \\
\hline & KLF9 & Hsa.41338 & $\begin{array}{l}\text { Human mRNA for GC box binding protein/ Kruppel Like } \\
\text { Factor } 9 \text {, complete cds }\end{array}$ & $\begin{array}{l}\text { Brown 2015, Ying 2014, } \\
\text { Simmen } 2008\end{array}$ \\
\hline & MEF2C & Hsa.5226 & $\begin{array}{c}\text { Myocyte-Specific Enhancer Factor 2, Isoform MEF2 } \\
\text { (Homosapiens) }\end{array}$ & $\begin{array}{l}\text { Chen 2017, Giorgio 2018, } \\
\text { Su2016 }\end{array}$ \\
\hline & GADPH & Hsa.1447 & Glyceraldehyde 3-Phosphate Dehydrogenase & Zhang 2015, Tang 2012 \\
\hline & TIMP3 & Hsa.11582 & Metalloproteinase Inhibitor 3 Precursor & Su 2019, Bai 2007 \\
\hline \multirow{8}{*}{ Leukemia } & TXN & X77584_at & TXN Thioredoxin & $\begin{array}{l}\text { Kamal 2016, Léveillard } \\
\text { 2017, Karlenius } 2010\end{array}$ \\
\hline & CSF3R & M59820_at & $\begin{array}{l}\text { CSF3R Colony stimulating factor } 3 \text { receptor } \\
\text { (granulocyte) }\end{array}$ & $\begin{array}{c}\text { Zhang 2018, Ritter 2020, } \\
\text { Klimiankou 2019, Lance } \\
2020\end{array}$ \\
\hline & MPO & M19508_xpt3_s_at & MPO from Human myeloperoxidase gene & $\begin{array}{c}\text { Szuber 2018, Kim 2012, } \\
\text { Lagunas-Rangel 2017, } \\
\text { Handschuh } 2019\end{array}$ \\
\hline & LYZ & M21119_s_at & LYZ Lysozyme & $\begin{array}{l}\text { Wang 2013, Liu 2018, } \\
\text { Tong } 2014\end{array}$ \\
\hline & CST3 & M27891_at & $\begin{array}{c}\text { CST3 Cystatin C (amyloid angiopathy and cerebral } \\
\text { hemorrhage) }\end{array}$ & Austin 2010 \\
\hline & ZYX & X95735_at & Zyxin & Austin 2010, Yunsong 2013 \\
\hline & CTSD & M63138_at & CTSD Cathepsin D (lysosomal aspartyl protease) & Wang2013 \\
\hline & $\begin{array}{l}\text { CD79A/ MB-1 } \\
\text { gene }\end{array}$ & U05259_rna1_at & MB-1 membrane glycoprotein & Wang 2013, Kozlov 2005 \\
\hline
\end{tabular}

3 
Figure 1

\section{Cluster Representative Refinement Procedure}

Each row of the table represents the gene with its class relevance value in terms of Pearson correlation coefficient with respect to sample class row. TR+ and TR- represent the augmented gene with their class relevance score in terms of Pearson correlation coefficient with respect to sample class row.

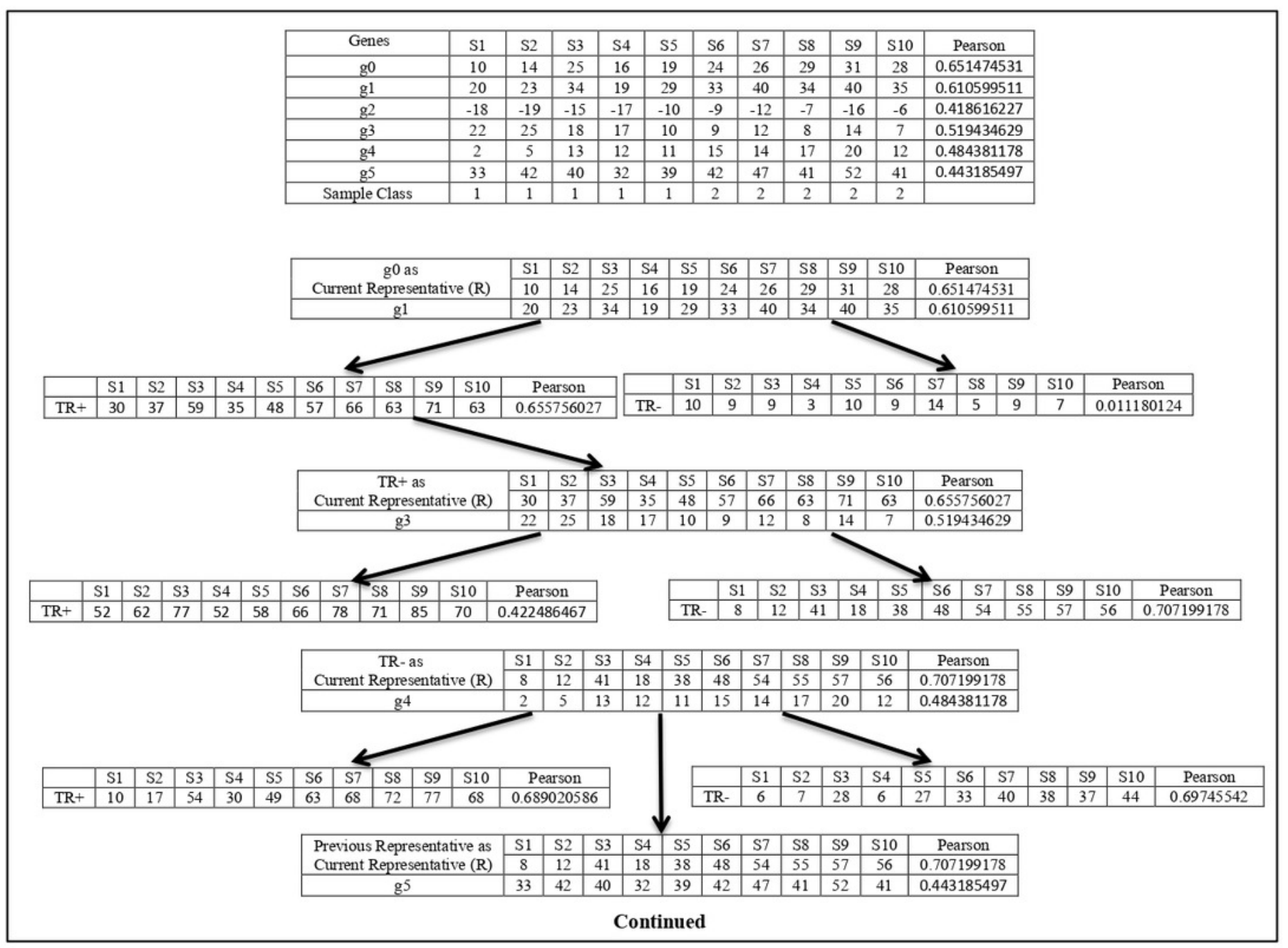


Figure 2

\section{Block Diagram of the Proposed MFSAC-EC Model}

Here $\mathrm{BK}_{1}, \mathrm{BK}_{2} \ldots . . \mathrm{BK}_{\mathrm{D}}$ are $\mathrm{D}$ number of bootstrapped datasets. $\mathrm{RSD}_{11} \ldots . \mathrm{RSD}_{17}$ represent different reduced sub datasets of $\mathrm{BK}_{1}$ bootstrapped datasets after applying MFSAC method. $I C_{11}$ to IC $\mathrm{IC}_{17}$ represent individual classifiers applied on $\mathrm{RSD}_{11} \ldots \mathrm{RSD}_{17}$ respectively.

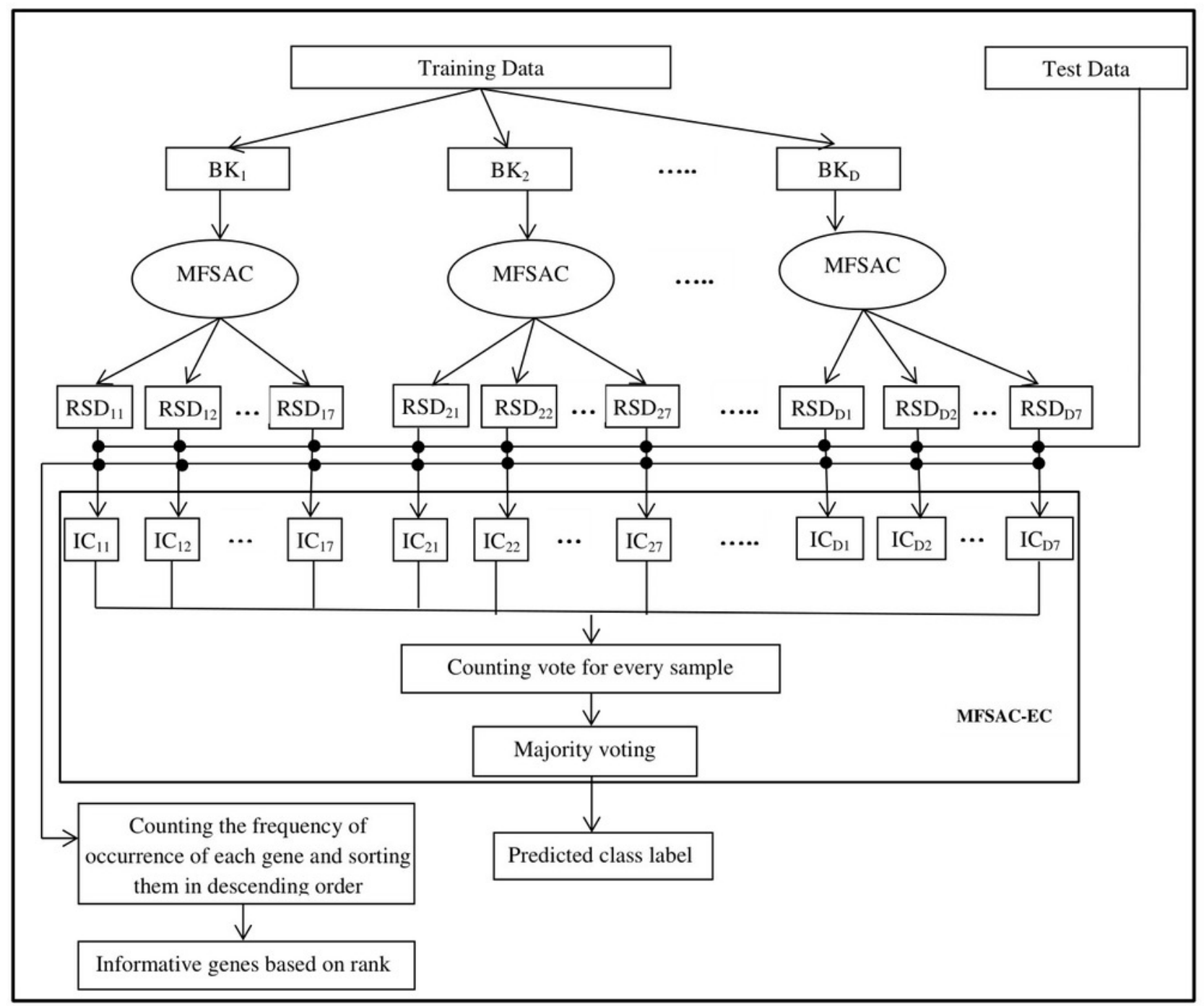


Figure 3

\section{Block Diagram of MFSAC Method}

$\mathrm{BK}_{1}$ is the Ith bootstrapped dataset. $\mathrm{FT}_{1} \ldots . . . \mathrm{FT}_{7}$ are the seven filter score functions as Supplemental Table $\mathrm{S1} \mathrm{SD}_{11} \ldots . \mathrm{SD}_{17}$ are sub datasets created after applying filter score functions. SAC is the Supervised attribute clustering method applied to generate $\mathrm{RSD}_{11} \ldots$. $\mathrm{RSD}_{17}$ reduced sub datasets.

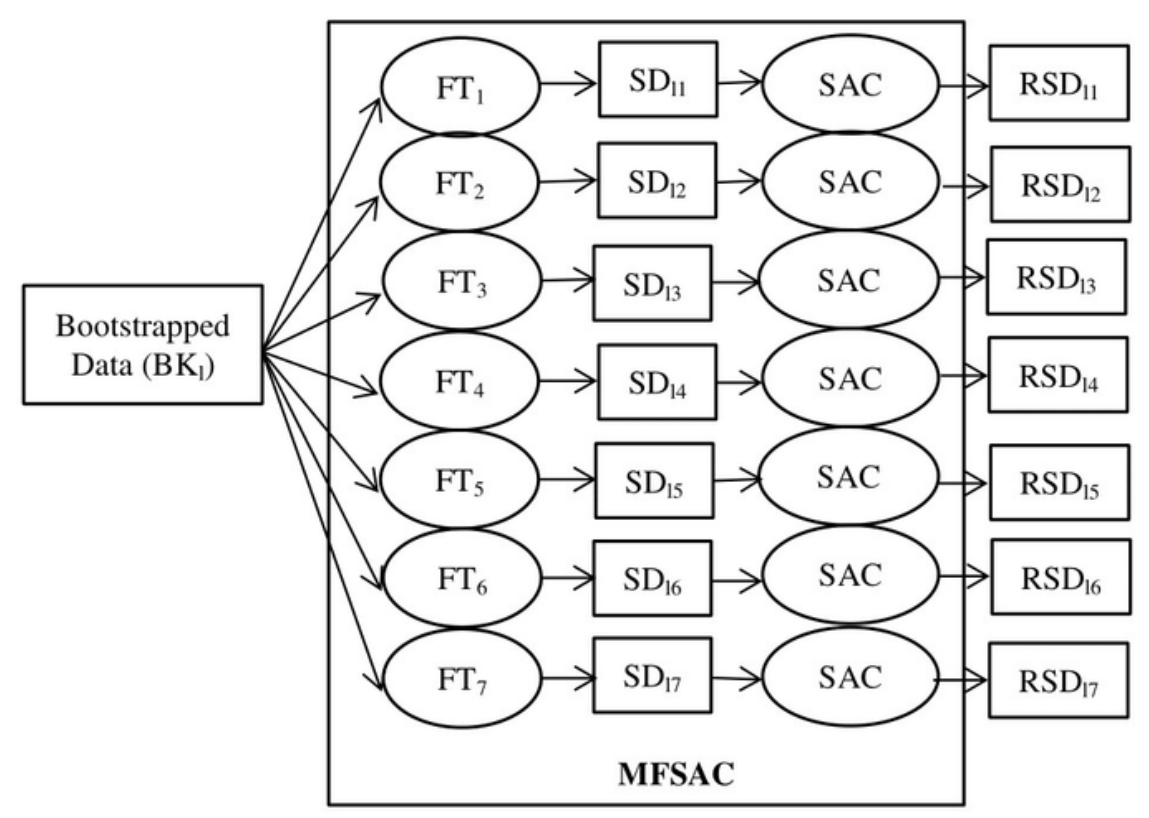


Figure 4

AUC for for three datasets using MFSAC-EC+ KNN, MFSAC-EC+NB, MFSAC-EC+ DT and MFSAC-EC+ SVM Classifiers.

(a) For Breast Cancer dataset using LOOCV. (b) For Colon Cancer dataset using 5-Fold Cross Validation. (c) For RAHC dataset using 10-Fold Cross-Validation
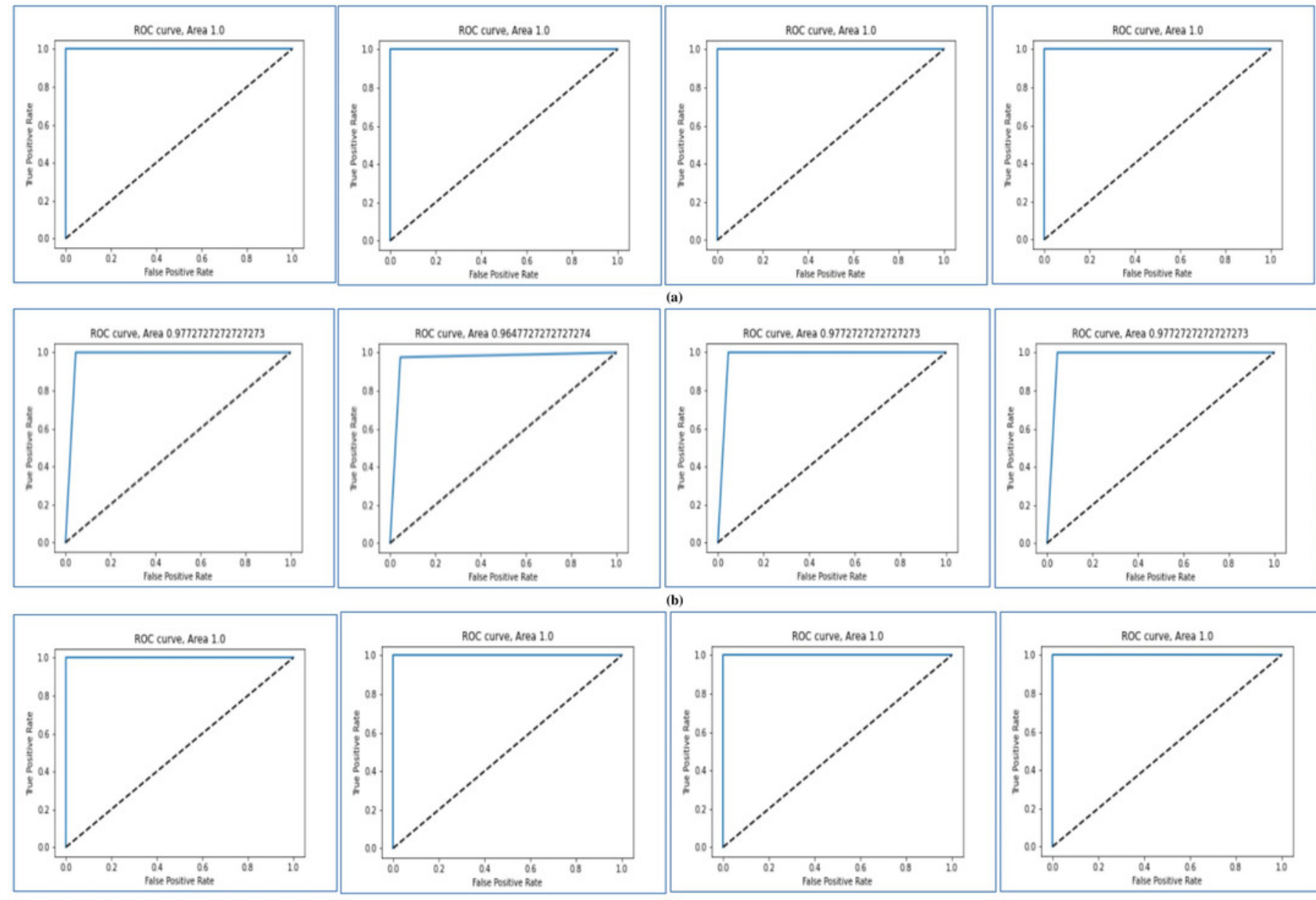


\section{Figure 5}

Heatmap of MFSAC-EC with base classifiers NB, KNN, DT and SVM respectively for multiclass datasets.

(a) For SRBCT dataset using 5-Fold Cross-validation. (b) For MLL dataset using 10-Fold CrossValidation.

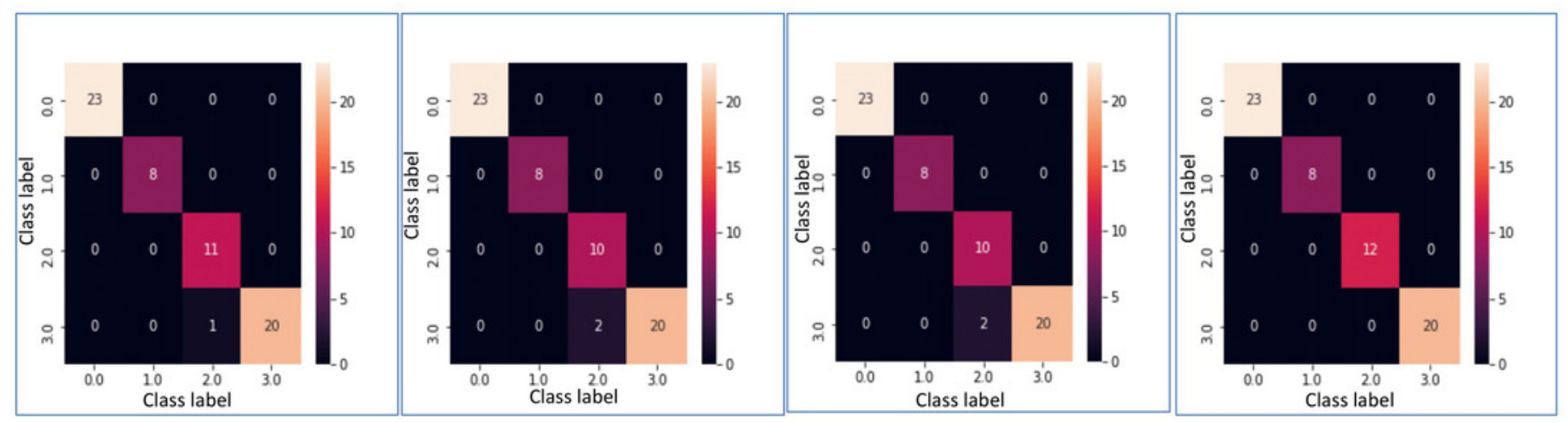

(a)

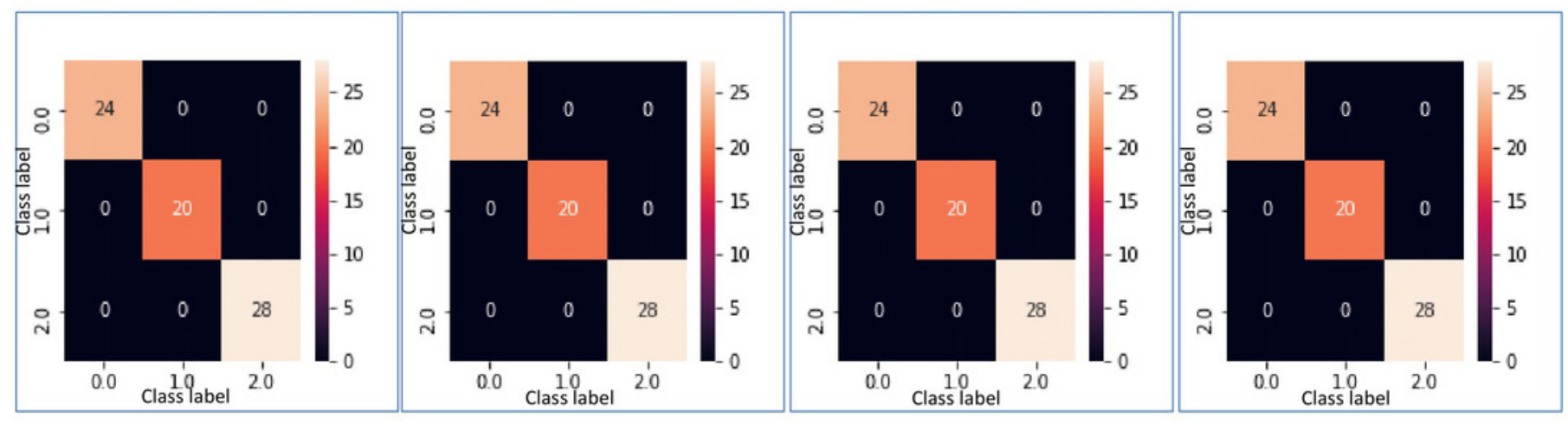

(b) 


\section{Figure 6}

Comparison of MFSAC-EC with other well-known supervised gene selection methods and full gene set in terms of 10-Fold Cross-Validation for all Datasets.

In each figure classification accuracy(\%) of MFSAC-EC model along with other supervised gene selection methods for all datasets are represented using different colored bars using (a) NB (b) KNN (c) DT and (d) SVM as base classifier.

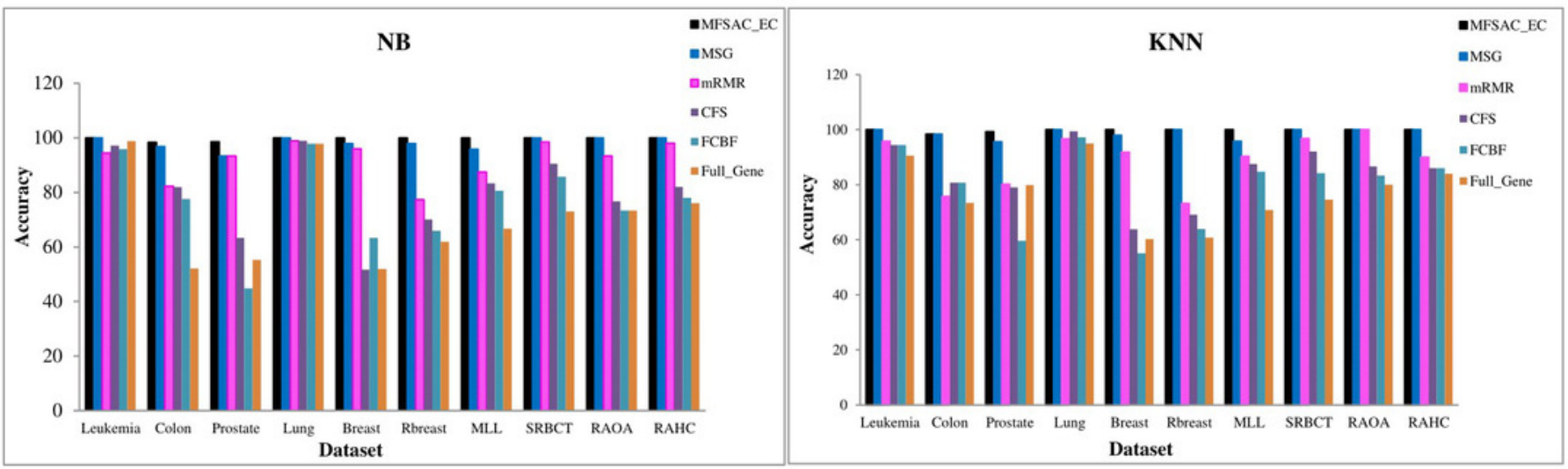

(a)

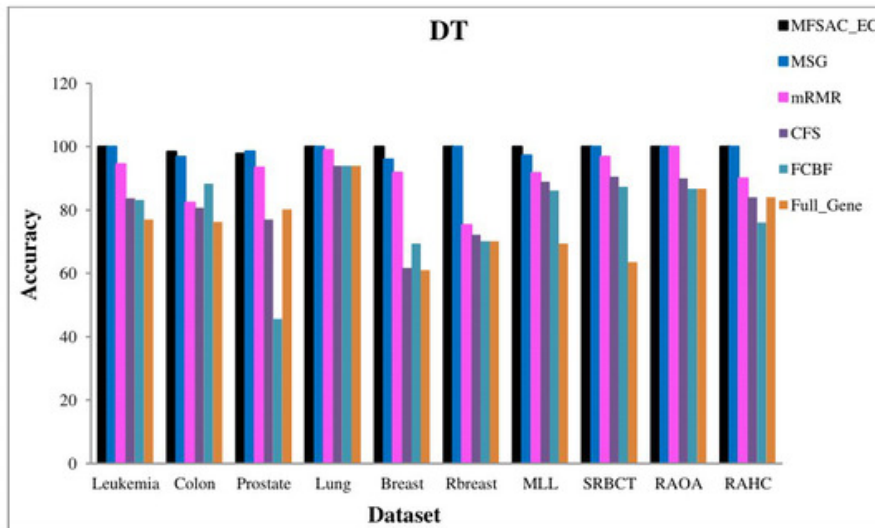

(b)

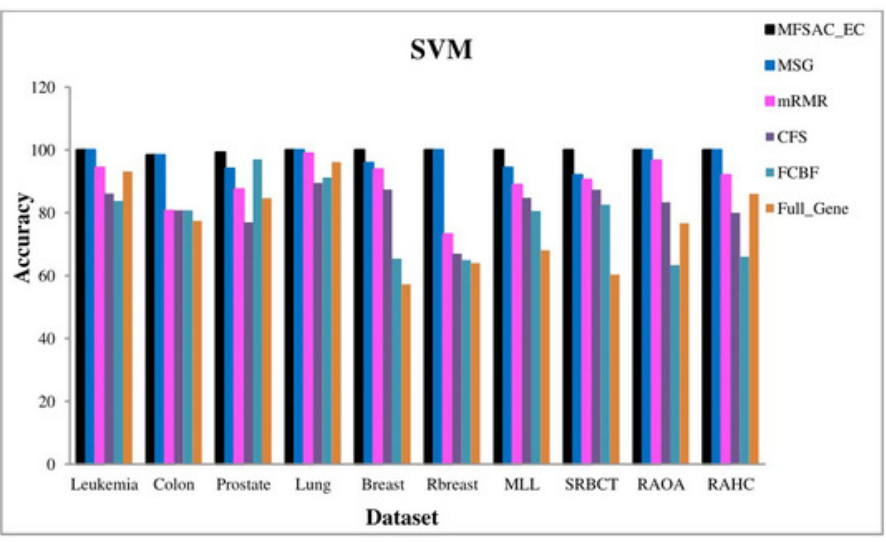

(c)

(d) 


\section{Figure 7}

Comparison of MFSAC-EC with other well-known unsupervised gene selection methods in terms of random splitting for different datasets.

In each figure classification accuracy(\%) of MFSAC-EC model along with other unsupervised gene selection methods for four datasets are represented with different colored bars using (a) NB (b) DT and (c) SVM as base classifier. 

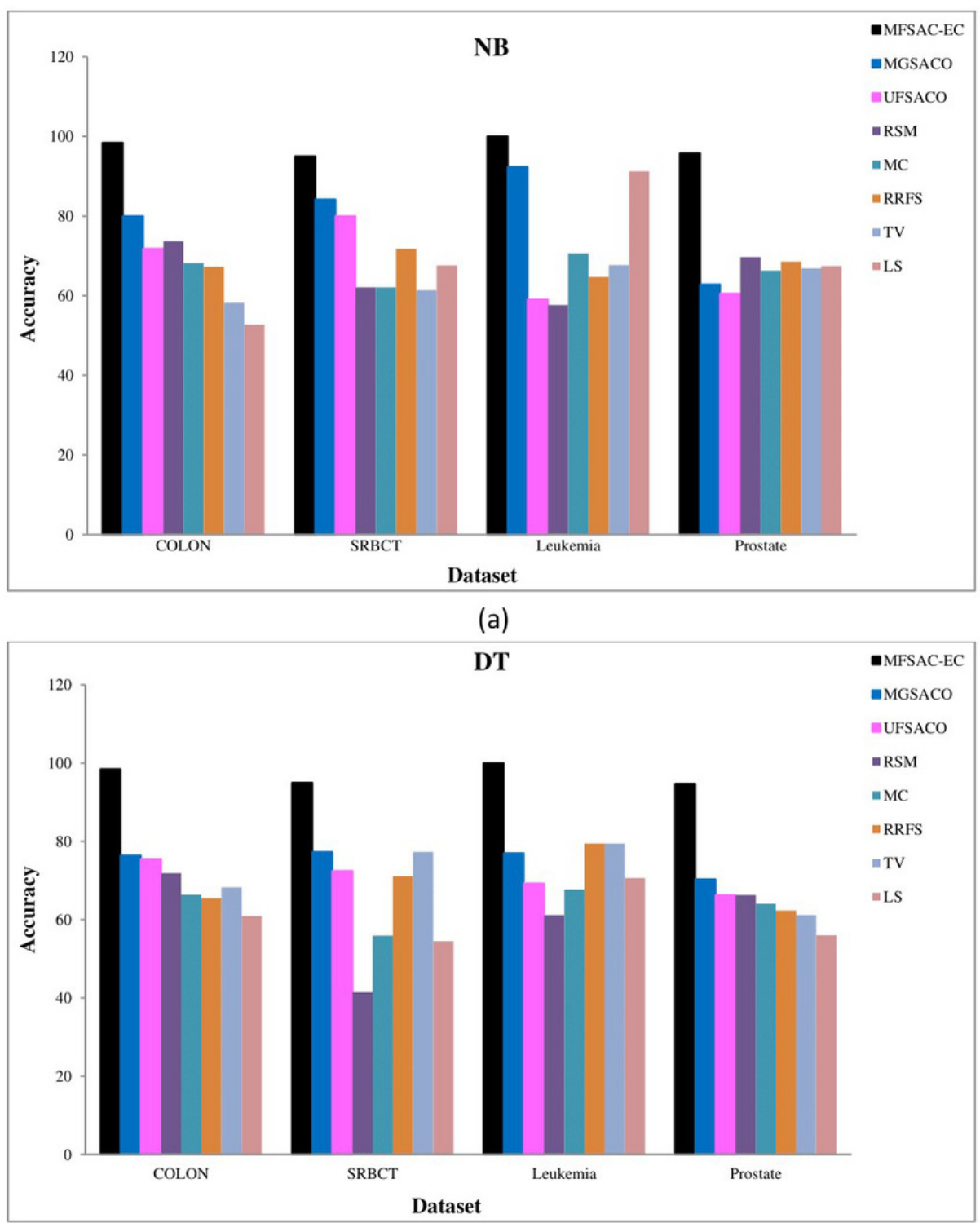

(b)

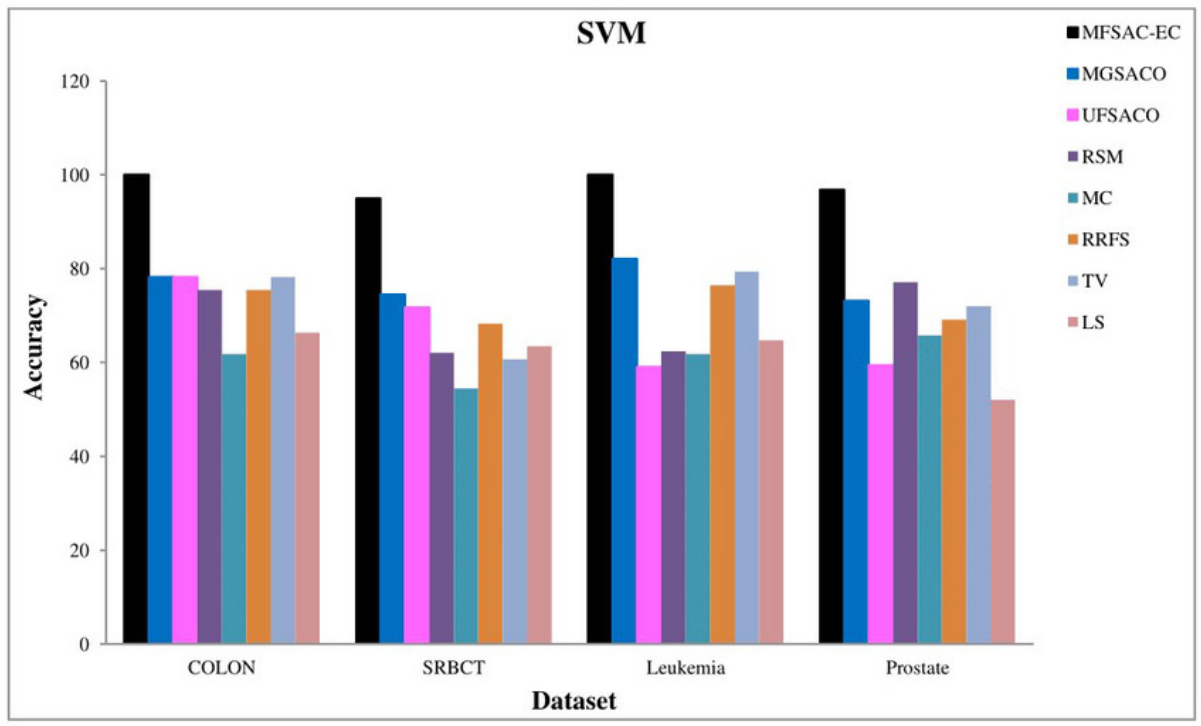

(c) 


\section{Figure 8}

Original gene (different class label with different color) and corresponding Augmented gene with respect to different filter methods for Breast Cancer dataset

Seven figures for seven different filter score function are shown here. In each figure the original gene and augmented gene are plotted with respect to sample class label. X-axis represents class label while $Y$-axis represents expression value. Two different class labels are represented by Blue and Red color. The difference of expression values of two classes in the augmented gene shows class discrimination ability of that gene. Gene number is the column number in the original dataset. 


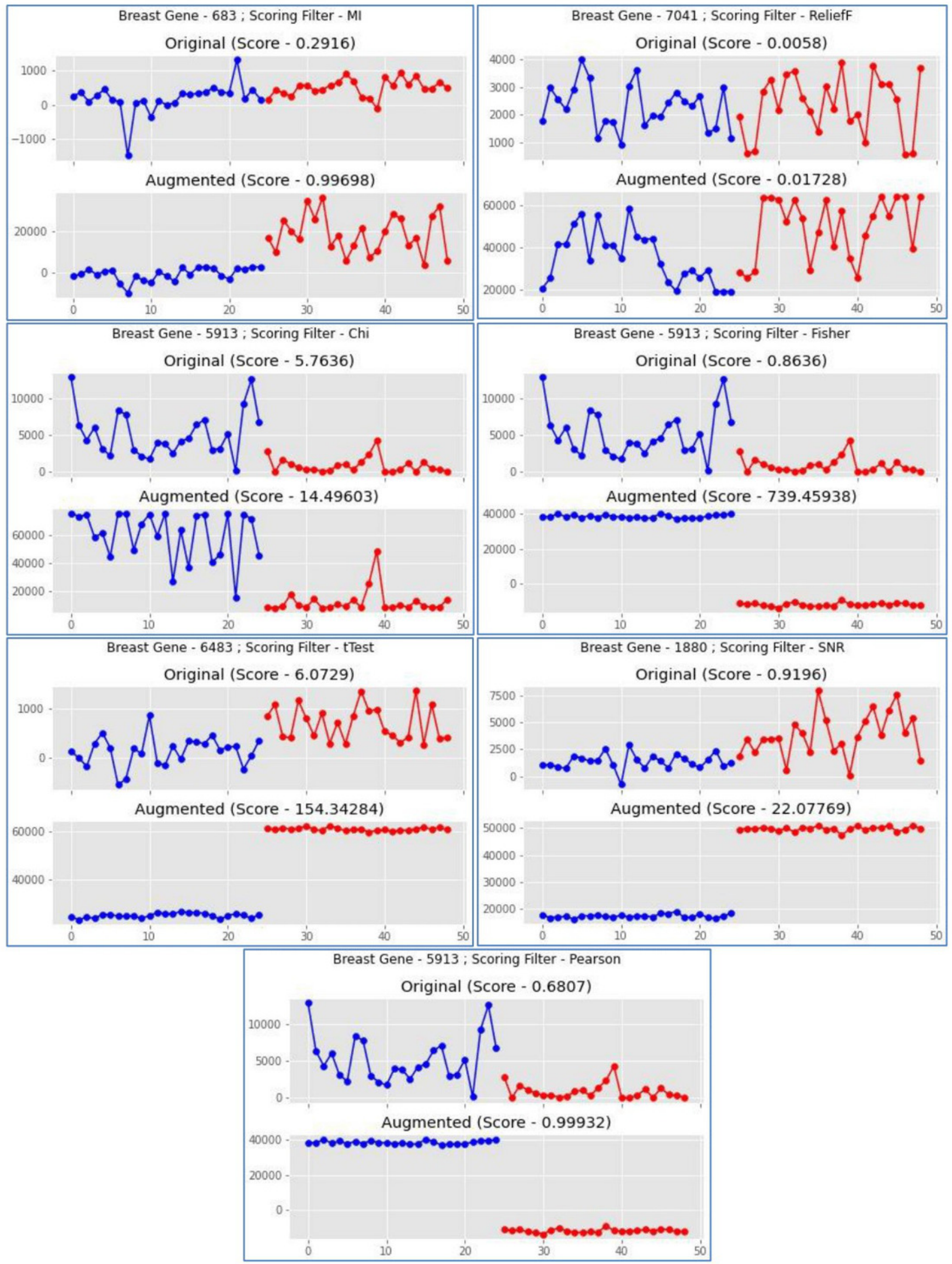

